\title{
Matter From Geometry Without Resolution
}

\author{
Antonella Grassi ${ }^{1}$, James Halverson ${ }^{2}$, and Julius L. Shaneson ${ }^{1}$ \\ ${ }^{1}$ Department of Mathematics, University of Pennsylvania \\ Philadelphia, PA 19104-6395 USA \\ ${ }^{2}$ Kavli Institute for Theoretical Physics, University of California \\ Santa Barbara, CA 93106-4030 USA
}

\begin{abstract}
We utilize the deformation theory of algebraic singularities to study charged matter in compactifications of M-theory, F-theory, and type IIa string theory on elliptically fibered Calabi-Yau manifolds. In Ftheory, this description is more physical than that of resolution. We describe how two-cycles can be identified and systematically studied after deformation. For ADE singularities, we realize non-trivial ADE representations as sublattices of $\mathbb{Z}^{N}$, where $N$ is the multiplicity of the codimension one singularity before deformation. We give a method for the determination of Picard-Lefschetz vanishing cycles in this context and utilize this method for one-parameter smooth deformations of ADE singularities. We give a general map from junctions to weights and demonstrate that Freudenthal's recursion formula applied to junctions correctly reproduces the structure of high-dimensional ADE representations, including the 126 of $S O(10)$ and the 43,758 of $E_{6}$. We identify the Weyl group action in some examples, and verify its order in others. We describe the codimension two localization of matter in F-theory in the case of heterotic duality or simple normal crossing and demonstrate the branching of adjoint representations. Finally, we demonstrate geometrically that deformations correctly reproduce the appearance of non-simply-laced algebras induced by monodromy around codimension two singularities, showing the reduction of $D_{4}$ to $G_{2}$ in an example. A companion mathematical paper will follow.
\end{abstract}




\section{Contents}

1 Introduction

2 From Deformations to Junctions: Basic Formalism 6

2.1 Singularities, Deformations, and Seven-Branes . . . . . . . . . . . . . 7

2.2 Matter Fields from Junctions and Two-Spheres . . . . . . . . . . . . . . . . . 8

2.3 An Interlude on $A_{r}$ Algebras $\ldots \ldots \ldots \ldots \ldots \ldots \ldots$

2.4 Topological Intersections Induce a Symmetric Bilinear Form . . . . . . . . . . . . . . . 10

2.5 Picard-Lefschetz Theory . . . . . . . . . . . . . . . . . . . . 11

3 Deformations and ADE Algebras 14

3.1 The Canonical Basis . . . . . . . . . . . . . . . . . . . . . . . . . . . 14

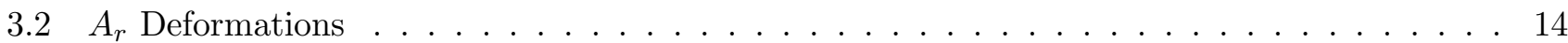

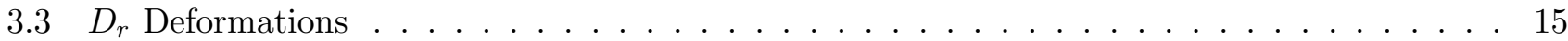

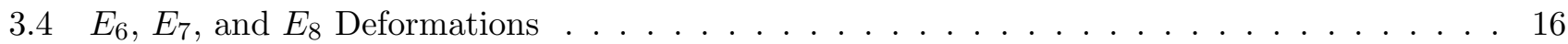

3.5 Non-trivial Representations and Freudenthal's Formula . . . . . . . . . . . . . . . 17

4 Massless Matter in Codimension Two 18

5 Illustrative Examples $\quad 21$

5.1 Matter from Branching Rules . . . . . . . . . . . . . . . . . . . . . 22

5.2 O-monodromy and Reducing $D_{4}$ to $G_{2} \ldots \ldots \ldots \ldots \ldots \ldots \ldots \ldots \ldots$

5.3 Matter Representations in Particle Physics . . . . . . . . . . . . . . 28

6 Conclusions and Future Directions $\quad 30$

A Appendices on ADE Algebras 31

B A Computational Package for Junctions $\quad 37$

\begin{tabular}{ll} 
C Explicit Junction Depiction & 38 \\
\hline
\end{tabular} 


\section{Introduction}

The spectrum of particles which exist in Nature is detailed and rich. The quarks and leptons of the standard model of particle physics fill out non-trivial representations of the Lie algebra $S U(3) \times S U(2) \times U(1)$, and these can be embedded into representations of higher rank groups, such as the $\mathbf{1 0}+\overline{\mathbf{5}}+\mathbf{1}$ of $S U(5)$ or the 16 of $S O(10)$. Exotic particle representations are often introduced in phenomenologically motivated extensions of the standard model, sometimes of high dimension in grand unified theories.

An important physical question is whether there exist theoretical constraints on the allowed particle representations. Though anomaly cancellation provides constraints on sets of fields in gauge theories, no individual representation is ruled out on theoretical grounds. By contrast, the possibilities 1 are more limited in four-dimensional compactifications of string theory, F-theory, and M-theory. For example, in the heterotic string matter representations typically arise from branching the adjoint of $E_{8}$; in weakly coupled type II string theory $U(N)$ gauge theories admit bifundamental, symmetric tensor, and antisymmetric tensor representations, together with their conjugates; in weakly coupled orientifold compactifications it is not possible to realize the phenomenologically relevant $\mathbf{1 6}$ of $S O(10)$.

Interestingly, more possibilities can be realized outside of the weakly coupled regime in M-theory [1] or F-theory [2]. In these theories massless matter representations are encoded in the structure of a singular compactification geometry, and the possibilities are broader than in the weakly coupled superstring theories. Studying matter in $d=4$ compactifications of M-theory requires a detailed knowledge of codimension seven singularities in compact singular $G_{2}$ manifolds, which, despite much progress in the construction of compact smooth $G_{2}$ manifolds [5] singular elliptically fibered Calabi-Yau varieties, and therefore compactifications of F-theory 2 on them may currently be the broadest framework for studying matter representations in the string landscape.

A great deal is already known [9-14] about the structure of matter in compactifications on singular elliptically fibered Calabi-Yau varieties with a section, namely their Weierstrass model. The resolution of generic singularities 3 produces a new singular elliptic fiber above the general point of the discriminant locus: it is a tree of holomorphic curves whose dual graph structure coincides with the extended Dynkin diagram of an ADE algebra $\mathfrak{g}$ of rank $r$. All massless W-bosons of $\mathfrak{g}$ appear by wrapping branes on these curves and taking a singular limit. In codimension two, singularity enhancement gives rise to matter representations of $\mathfrak{g}$. If the enhancement is of a simple type to another ADE algebra $\mathfrak{g}^{\prime}$ of rank $r+1$, the representation of the localized matter 4 can be determined 9 , by the branching rules of the adjoint of $\mathfrak{g}^{\prime}$; there exist more exotic possibilities [11,13,14] at higher codimension in moduli space. In addition, monodromy around codimension two loci can induce an outer automorphism on $\mathfrak{g}$ which reduces it [10] to a non-simply laced gauge algebra $\mathfrak{g}^{\prime}$. We refer to this as outer monodromy or O-monodromy.

Resolution has been an important tool for understanding other aspects of physics in many recent works. This is particularly true in F-theory, which has received a great deal of attention in the last years, initiated by work [32,33] on grand unified models. Since then, there has been significant progress in understanding F-theory compactifications to four dimensions, including globally consistent models [34 49],

\footnotetext{
${ }^{1}$ See 3, 4] for a systematic study of constraints on matter representations in the free field heterotic string.

${ }^{2}$ Though we may use the language of F-theory throughout this paper, our results also apply in other contexts, as they are ultimately results about the homology of elliptic fibrations.

${ }^{3}$ That is, a smooth point of the codimension one locus of the discriminant.

${ }^{4}$ Ideas from both singularity resolution and deformation were used in 9 ].
} 
$U(1)$ symmetries [50 56], instanton corrections [42, 52, 57 64], and chirality inducing $G_{4}$ - $f$ lux [52, 64 76]; there has also been progress in understanding the landscape of six-dimensional F-theory compactifications [54, 77 80]. Singularity resolution was utilized heavily in many of these works. While the resolution of generic singularities is well understood and goes back to Kodaira's work, the resolutions in higher codimensions have to be worked out case by case and can be rather complicated, see for example [81.

Since four-dimensional $\mathcal{N}=1$ compactifications of F-theory provide an enormous class of vacua, continued progress in this direction is critical for our understanding of the landscape. Studying the physics of these compactifications via resolution amounts to studying the Coulomb branch of the defining three-dimensional $\mathcal{N}=2$ M-theory compactification. While this approach is useful in many cases - for example Chern-Simons terms in three dimensions beautifully encode the structure of four-dimensional gauge anomalies [82] — it is rather indirect: the Coulomb branch does not exist in the F-theory limit. It would be better, when possible, to study F-theory by methods which exist in both the defining M-theory compactification and also in the F-theory limit. This work is a step in that direction.

We study the appearance of vector and matter multiplets in non-trivial Lie algebra representations via the deformation theory of algebraic singularities in elliptically fibered varieties $X$; see [29, 30, for a study in heterotic M-theory. This involves movement in the complex structure moduli space of $X$, rather than the Kähler moduli space of resolution. Though for M-theory on $X$ deformation and resolution are complimentary approaches for understanding gauge theoretic structure, only the deformation picture is physical in the F-theory limit. For example, ADE states which obtain mass upon Higgsing the gauge theory arise from two-cycles whose non-zero volume is obtained from deformation, not resolution. This picture is also necessitated by heterotic duality [2, 18, 19]: at a generic point in the moduli space of an $E_{8}$ gauge bundle the theory is completely Higgsed and the $240 \mathrm{~W}$-bosons of $E_{8}$ are massive; in the dual F-theory picture the heterotic bundle moduli map to complex structure moduli which deform the $E_{8}$ singularity and give rise to the necessary two-cycles. Klein showed that resolutions and deformation of ADE surface singularities (also known as kleinan singularities) are diffeomorphic. We use deformation and junctions to naturally associate weights and other representations, in addition to the adjoint to these singularities.

It is simple to see how deformation differs from resolution, and why it is physically relevant. Consider an elliptic fibration $5 \stackrel{\pi}{\rightarrow} B$ with discriminant $\Delta$. If one of the components of the discriminant is smooth, or locally smooth, it can be described by $z=0$ and the discriminant takes the form $\Delta=z^{N} \tilde{\Delta}$, where $\tilde{\Delta}$ is a residual piece which can be computed in examples when $X$ is a Weierstrass model. If $N=1$, then the Weierstrass model $X$ is generically smooth along $z=0$ and the theory there is completely Higgsed; if $N \geq 2$ generically $X$ has ADE singularities with algebra $\mathfrak{g}$ along $z=0$, and performing a small deformation of the elliptic fibration such that the theory is completely Higgsed gives

$$
\Delta \sim z^{N} \stackrel{\text { deform }}{\longrightarrow} \quad \Delta \sim \prod_{i=1}^{N}\left(z-\varepsilon_{i}\right)
$$

in a general neighborhood of $z=0$. We emphasize that $N$ is not 6 the rank of $\mathfrak{g}$. In the language of Ftheory it is the number of seven-branes, and therefore $N$, and not just $r$, must play a role in determining

\footnotetext{
${ }^{5}$ Note that $X$ could be, but is not required to be, a Calabi-Yau variety. Sometimes we utilize subscripts to denote the complex dimension of these spaces as $X_{d}$ and $B_{d-1}$. We will often consider $X$ in Weierstrass form.

${ }^{6}$ The cases $G=A_{r}$ or $G=D_{r}$ satisfy $N=r+1$ and $N=r+2$, respectively; $E_{6} E_{7}$ and $E_{8}$ satisfy $N=r+2$.
} 
the structure of ADE states which end on seven-branes.

We demonstrate that the Lie-algebraic structure of these states is determined by two-cycles arising from the deformation of ADE singularities, which can be specified as vectors in $\mathbb{Z}^{N}$. Together with an appropriate inner product, the two-cycles which naturally arise from deformation give rise to root lattices which span $r$-dimensional subspaces of $\mathbb{Z}^{N}$. We emphasize that these deformation techniques and string junctions can be applied to codimension two singularities in Weierstrass models to detect matter and non-simply laced algebras; in section 5.5 we study the algebra $\mathfrak{g}_{2}$ via deformation and string junctions. That is, we study matter without resolution.

We reproduce the IIb string junction formalism of [15]17]; as such, we will refer to these two-cycles as junctions. There is naturally some overlap between the group theoretic results we obtain and the results of [15], though we generalize the group theory and emphasize the relationship to deformation theory and geometry. We will attempt to distinguish new results from old, when possible. We also emphasize that the formalism of junctions is computationally much simpler than that of singularity resolution; in appendix B we provide a publicly available software package for performing these computations. In the companion paper [20] we analyze the mathematical underpinning of matter without resolution.

Let us summarize our results and the outline of this paper.

In section 2 we lay out the basic formalism for understanding two-cycles and deformations. The input data for analysis is an ordered set $Z$ of vanishing one-cycles in the elliptic fiber, obtained from geometry after singularity deformation. Two-cycles arise naturally from this data, and for particular sets $Z$ the $A_{r}$ algebras arise intuitively. More generically, topological self-intersections induce a symmetric bilinear form $I$ which becomes the product on the root systems in the case of deformations of ADE singularities. For a given set $Z$ this product can be represented as an $N \times N$ matrix. The general setup admits a description in terms of Picard-Lefschetz monodromy and the related theory for elliptic surfaces. The choice of $Z$ amounts to the choice of a strong basis [23], and the symmetric bilinear form on the algebra is independent of this choice. We give a method for the explicit determination of $Z$, which we exemplify later in the paper.

In section 3 we specialize to the case of $\mathrm{ADE}$ deformations and representations. We review the socalled canonical presentation of [15], which which gives a set $Z$ for each ADE group. Our general formula for the symmetric bilinear form reproduces the known results for ADE deformations in the canonical presentation. For each ADE group, we specify a surface deformation which completely Higgses the gauge group. We determine ordered sets $Z$ associated to these deformations, which differ from the results of [15]; nevertheless a junction analysis can be performed and the representation theoretic results are consistent.

We discuss the appearance of non-trivial representations as junctions with non-zero asymptotic charge. These are real two-manifolds emanating from a complex codimension one locus with associated algebra $\mathfrak{g}$; studying the $\mathfrak{g}$-representation theory only requires knowledge of codimension one data. Utilizing the symmetric bilinear form $I$, we construct a generic map from junctions to weights in the Dynkin basis and demonstrate that Freudenthal's recursion formula also applies to junctions, using $I$ as the product. This is essential since it allows for the study of junctions in representations $\rho$ of $\mathfrak{g}$ with non-trivial weight multiplicities and lengths; moreover, it is necessary for isomorphism. As a non-trivial check, we have verified that the junction representations of the $\mathbf{1 2 6}$ of $S O(10)$ and the $\mathbf{4 3 , 7 5 8}$ of $E_{6}$ appear in the expected way. 
In section 4 we discuss the appearance of massless matter in codimension two at $z=t=0$. As emphasized, the representation theory is determined explicitly by codimension one data, but the codimension two data is critical for determining which representations become massless. We focus on the cases where $X$ is $K 3$-fibered or the codimension two singularity arises from simple normal crossing; in these cases the localization of matter in codimension two can be understood by studying families of deformed elliptic surfaces parameterized by $t$. In some cases the matter localized in codimension two follows from branching junctions in the adjoint. We argue that in the deformation picture O-monodromy around codimension two loci can induce an outer automorphism on junctions which reduces $\mathfrak{g}$ to a non-simply laced algebra $\mathfrak{g}^{\prime}$.

In section [5 we provide a number of illustrative examples with non-trivial behavior in codimension two. Via explicit deformation we determine matter from the branching of adjoints for $A_{r} \rightarrow A_{r+1}$ enhancement; in the canonical basis we demonstrate the branching of the adjoints of $E_{6}$ and $S O(8)$ to the 16 of $S O(10)$ and the 6 of $S U(4)$, describing weak coupling interpretations when possible. We explicitly demonstrate that O-monodromy can be studied and understood in the deformation picture. Specifically, we deform a $D_{4}$ singularity with appropriate codimension two structure and demonstrate that O-monodromy induces an outer automorphism on the $D_{4}$ roots, represented as junctions, that reduces $D_{4}$ to $G_{2}$. The basis we determine for these deformations differs from the canonical basis and makes the $\mathbb{Z}_{3}$ automorphism more transparent; a similar computation was performed in the canonical basis in [31], though the results were not derived directly from a deformed geometry. We also discuss junction realizations of ADE representations which play important roles in particle physics models.

\section{From Deformations to Junctions: Basic Formalism}

In this section we will lay out formalism in a general manner which includes, but is not limited, the case of deformations of ADE singularities. We begin by discussing an elliptic fibration $X$ and an associated ordered set of vanishing one-cycles $Z$. The fiber above a component $\Delta_{i}$ of the discriminant $\Delta$ of $X$ has a vanishing one-cycle $\pi_{i}$. Two components of the discriminant can be connected by strings; more generically $n$-components can be connected by an $n$-pronged "string junction." Such an object gives rise to a twomanifold in the total space $X$ and can be represented as a vector in $\mathbb{Z}^{N}$. In a neighborhood of nearby components $\Delta_{i}$ we define a product on these vectors which amounts to the topological self-intersection of two-cycles. This induces a symmetric bilinear form on $\mathbb{Z}^{N}$ which becomes the product on the ADE root system for ADE deformations. This formalism admits a description in terms of Picard-Lefschetz theory. We give a method for determining $Z$. 

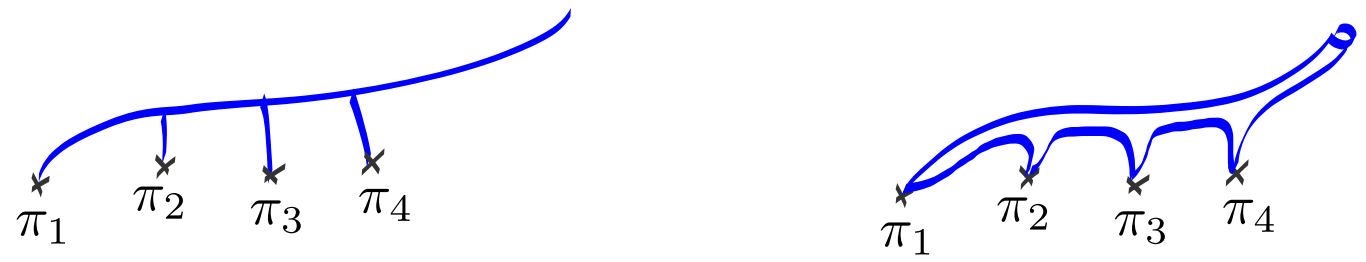

Figure 1: On the left is a four-pronged junction in $B$. The picture on the right is of the corresponding real two-manifold in the total space $X$. The loose end wraps the one-cycle $a(J)$ in the elliptic fiber; i.e. the asymptotic charge.

\subsection{Singularities, Deformations, and Seven-Branes}

Consider the elliptic fibration $X$ and consider also the case where there are $k \geq 2$ components of the discriminant. Fix a base point $P$ on $B \backslash \Delta$ and a basis for $H_{1}$ (fiber). Above each component $\Delta_{i}$ of the discriminant is an elliptic fiber with a vanishing one-cycle $\pi_{i}$; after choosing a path in $B \backslash \Delta$ which approaches $\Delta_{i}$, together with generators of one-cycles in the fiber above $P$, we may write $\pi_{i}=\left(\begin{array}{c}p_{i} \\ q_{i}\end{array}\right) \in \mathbb{Z}^{2}$. In an F-theory compactification, such a geometry gives a $\left(p_{i}, q_{i}\right)$ seven-brane along $\Delta_{i}=0$. For any geometry it is critical to identify the components of the discriminant, their vanishing cycles, and a choice of path around them; this determines an ordered set $Z$ of vanishing cycles which serves as input data for studying two-cycles. We will see that this is sufficient to begin uncovering Lie algebraic structure and leave a detailed discussion of monodromy and the determination of $Z$ until section 2.5.

As a brief aside, it is useful to review how well-known physical scenarios can be described in terms of $(p, q)$ seven-branes, and also to set conventions 7 which will be useful in discussing deformations of ADE singularities and their associated algebras. The only seven-branes which exist in the weakly coupled type IIb limit 83, 84 are D7-branes and O7-planes, and we follow the convention in the literature where a D7-brane is called an $A$-brane and has $\pi_{A} \equiv\left(\begin{array}{l}1 \\ 0\end{array}\right)$. In F-theory the string coupling $g_{s}$ is finite and $\mathrm{D}(-1)$-instanton effects split an O7-plane into a $B$-brane and a $C$-brane, which have $\pi_{B}=\left(\begin{array}{c}1 \\ -1\end{array}\right)$ and $\pi_{C}=\left(\begin{array}{l}1 \\ 1\end{array}\right)$, respectively. This splitting is precisely equivalent to the quantum splitting due to instantons in Seiberg-Witten theory [85], where the $\mathcal{N}=2 d=4$ gauge theory with $G=S U(2)$ is the worldvolume gauge theory of a D3-brane probing the local $B C$-geometry [86; the gauge instantons of Seiberg-Witten theory are the D(-1)-instantons inside the D3 probe 8 . Singularities at codimension one in the base are classified by Kodaira and are ADE singularities for minimal Weierstrass models; after deformation these admit descriptions in terms of particular $A$-branes, $B$-branes, and $C$-branes. See section 3 .

The physical importance of deformation theory enters in a simple way. Let $N_{i} \equiv \operatorname{deg}\left(\Delta_{i}\right)$ be the degree of the component $\Delta_{i}$, so that we write $\Delta=\prod_{i} \Delta_{i}^{N_{i}}$. In any given example, only a certain set of deformations are consistent with the elliptic fibration $X$. In performing the deformation of the geometry, the discriminant is changed such that the component $\Delta_{i}$ will deform into some number of other components. In the case of $\mathrm{ADE}$ singularities the relevant deformations are known and the degree $N$ component $\Delta_{z}$ splits into $N$ components, each of which has an elliptic fiber above it with a particular vanishing cycle.

\footnotetext{
${ }^{7}$ When possible, we follow the conventions of [15].

${ }^{8}$ These effects play an important role in understanding certain globally consistent F-theory compactifications [42].
} 


\subsection{Matter Fields from Junctions and Two-Spheres}

Armed with the knowledge of the components $\Delta_{i}$ and their vanishing cycles $\pi_{i}$, it is possible to study two-cycles which arise in the geometry. Physical objects, such as M2-branes, can wrap these two-cycles and give rise to representations $\rho$ of the gauge algebra $\mathfrak{g}$. In F-theory these states must be described as $n$-pronged string junctions in $B$, since only the dimensions of $B$ in the total space $X$ are physical.

Consider first two separated components $\Delta_{1}$ and $\Delta_{2}$ of the discriminant with vanishing one-cycles $\pi_{1}=\pi_{2}$. An oriented interval $J$ exists from $\Delta_{1}$ to $\Delta_{2}$; in the fiber above $\Delta_{1}$ the cycle $\pi_{1}$ vanishes, but upon moving along $J$ towards $\Delta_{2}$ it grows and then vanishes again at $\Delta_{2}$, giving a two-sphere with two marked points. Though $J$ is an interval in $B$ represented by the "brane charge" vector $(1,-1)$, denoting the interval orientation from $\Delta_{1}$ to $\Delta_{2}$, we see that it is also two-sphere in $X$, since $1 \times \pi_{1}+(-1) \times \pi_{2}=\left(\begin{array}{c}0 \\ 0\end{array}\right)$. More generically, one could consider $N$ components $\Delta_{i}$ connected in $B$ by an $n$-pronged object represented by the vector $J=\left(J_{1}, \ldots, J_{N}\right) \in \mathbb{Z}^{N}$ with $n$ of the $J_{i} \neq 0$. Such a $J$ is a junction or string junction. If $\sum_{i} J_{i} \pi_{i}=\left(\begin{array}{l}0 \\ 0\end{array}\right)$, this gives a two-sphere in the total space $X$ with $n$ marked points.

Thus far we considered a general set of components $\Delta_{i}$ and the associated two-cycles. It is possible, of course, that there is a natural grouping of components into multiple sets according to proximity to certain codimension one loci. For example, if a geometry realizes an $A_{r_{1}}$ and a $D_{r_{2}}$ singularity along $\Delta_{A}$ and $\Delta_{D}$, a generic but small deformation of the geometry deforms $\Delta_{A}$ into $r_{1}+1$ nearby components, and similarly deforms $\Delta_{D}$ into $r_{2}+2$ nearby components. As one might expect, the structure of appropriate two-spheres constructed within one set of components encodes the data of massive W-bosons, which become massless in codimension one upon undoing the deformation.

More generically, consider two sets $\Delta_{i}$ and $\Delta_{j}$ with $N_{1}$ and $N_{2}$ components and two-spheres which end on some number of components in each set. These could be represented by a vector in $J_{\text {tot }} \in \mathbb{Z}^{N_{1}+N_{2}}$. However, this isn't necessary for some purposes, since any representations arising from the $N_{1}$ components should be determined only by how a junction ends on those components. Truncating appropriately, we represent the junction prongs ending on the $N_{1}$ components by a vector $J=\left(J_{1}, \cdots, J_{N_{1}}\right) \in \mathbb{Z}^{N_{1}}$ with $\sum_{i} J_{i} \pi_{i} \neq\left(\begin{array}{l}0 \\ 0\end{array}\right)$, since the two-sphere determined by $J_{t o t}$ ends on some number of components in $\Delta_{j}$. For determining which set of components in $\Delta_{j}$ the junction $J$ emanating from $\Delta_{i}$ can end on, it is important to keep track of the asymptotic charge $a(J) \equiv \sum_{i} J_{i} \pi_{i} \equiv\left(\begin{array}{c}p_{J} \\ q_{J}\end{array}\right)$. In type IIb language, the corresponding state has $p_{J}$ units of $B$-field charge and $q_{J}$ units of Ramond-Ramond $C_{2}$ charge. For example, the junctions $J$ giving fundamentals of $S U(N)$ all have $a(J)=\left(\begin{array}{l}1 \\ 0\end{array}\right)$, which signifies that they can end on $D 7$-branes in codimension two. Antisymmetric tensors, on the other hand, have $a(J)=\left(\begin{array}{l}2 \\ 0\end{array}\right)$, recovering the fact that they become massless at intersections with $O 7$-planes.

In summary, we will represent junctions $J$ which emanate from a set of $N$ components $\Delta_{i}$ near a common codimension one locus by a vector $J \in \mathbb{Z}^{N}$, recognizing that most $J$ will have asymptotic charge. The ordered set of vanishing cycles $Z$ naturally determines a basis for $\mathbb{Z}^{N}$, the "junction basis." Whether a particular state $J$ becomes massless at a given codimension two locus depends on the asymptotic charge $a(J)$ and the vanishing cycles $\pi_{j}$ of the components $\Delta_{j}$; if the conditions on these quantities are appropriate, there will be vanishing two-cycles in codimension two. This will become clear in examples.

We refer the reader to appendix $\mathbb{C}$ for a detailed depiction of a non-trivial junction. 


\subsection{An Interlude on $A_{r}$ Algebras}

Though more generic cases will require further thought, the discussion thus far is sufficient for showing that the structure of $A_{r}$ algebras arises from deformation theory and junctions. It is our hope that this builds geometric and physical intuition before moving to the more complicated $D_{r}$ and $E_{6,7,8}$ cases.

Suppose the the local geometry of $X$ has an $A_{r}$ singularity along $\Delta_{z}$. In this case $N=r+1$ and we have $\Delta=z^{r+1} \tilde{\Delta}$, where $\tilde{\Delta}$ is a residual piece which would exist if this were a local description in a globally defined geometry. After performing the one-parametel 9 deformation (3.3), one arrives at $\Delta=\tilde{\Delta} \prod_{i=1}^{r+1}\left(z_{i}-\varepsilon_{i}\right) \equiv \tilde{\Delta} \prod_{i=1}^{r+1} \Delta_{i}$ where $\varepsilon_{i}$ are appropriate roots of the deformation parameter $\varepsilon$. For this geometry it is known 10 that $\pi_{1}=\cdots=\pi_{r+1} \equiv \pi$ for all components $\Delta_{i}$, and via an $S L(2, \mathbb{Z})$ transformation we can choose $\pi=\left(\begin{array}{l}1 \\ 0\end{array}\right)$ without loss of generality. A junction $J$ is determined by how it ends on the $r+1$ components $\Delta_{i}$, and therefore can be represented as a vector in $\mathbb{Z}^{r+1}$.

The $A_{r}$ algebra emerges from this data immediately. The $r+1$ components $\Delta_{i}$ are arranged on a circle around $z=0$, as drawn below, and the separation between the $\Delta_{i}$ is determined by the deformation parameter $\varepsilon$. Pick an ordered set $Z$ of the $\Delta_{i}$ by picking one to be the first element in $Z$ and choosing a direction around the circle. Consider two-cycles that occur in the geometry, utilizing an orthonormal basis $e_{i}$ for $\mathbb{Z}^{r+1}$ for convenience and defining the junctions $J_{i j} \equiv e_{i}-e_{j}$ with $i \neq j$. These satisfy $a\left(J_{i j}\right)=0$ and give two-spheres with two marked points in $X$. Let us briefly consider $A_{4}$ for the sake of visualization. A useful picture is

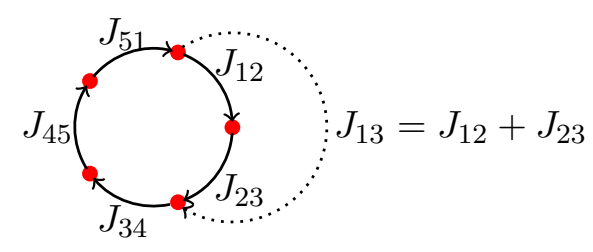

where the red dots are the components $\Delta_{i}$ and the arrows depict some junctions, which are one-dimensional strings in $B$ but are two-spheres in $X . J_{51}=-J_{12}-J_{23}-J_{34}-J_{45}$ is not an independent junction, and $J_{13}=J_{12}+J_{23}$ where the addition is in the second homology. For general $r$, there are $(r+1)^{2}-r-1 J_{i j}$ with $i \neq j$, which also happens to be the number of the roots of $A_{r}$. Defining $\alpha_{i} \equiv J_{i, i+1}$, we see that $\alpha_{1}$ and $\alpha_{2}$ can be added to give $J_{1,3}=\alpha_{1}+\alpha_{2}$, as depicted above. Letting $(\cdot, \cdot)$ be the negative of the standard inner product on $\mathbb{Z}^{r+1}, A_{i j} \equiv\left(\alpha_{i}, \alpha_{j}\right)$ is the negative Cartan matrix of $A_{r}$. This is in accord with standard convention in algebraic geometry, where simple roots obtained by resolution are $(-2)$-curves. Though we have arrived at the roots $J_{i j}$ intuitively, one could have also found them by searching for all $J$ with $(J, J)=-2$ and vanishing asymptotic charge. Similarly, solving for $J$ with $(J, J)=-1$ and $a(J)=\left(\begin{array}{l}1 \\ 0\end{array}\right)$ or $a(J)=\left(\begin{array}{c}-1 \\ 0\end{array}\right)$ give the fundamental and antifundamental, respectively. $J$ with $(J, J)=-1$ and $a(J)=\left(\begin{array}{l}2 \\ 0\end{array}\right)$ gives the antisymmetric tensor representation.

We considered a one parameter deformation of the $A_{r}$ singularity which completely Higgsed the gauge group. More generically, there are $r$ inequivalent deformations, which can be written naturally in a Weylinvariant form [21]. The Weyl group $S_{r+1}$ permutes the $r+1$ defects.

\footnotetext{
${ }^{9}$ Deformations with more parameters exist; we study one-parameter deformations which completely Higgs the gauge group.

${ }^{10}$ This will be explicitly derived later.
} 


\subsection{Topological Intersections Induce a Symmetric Bilinear Form}

In section 2.3 the product $(\cdot, \cdot)$ played an important role in determining roots and weights. In the elliptic surface defined by a one-dimensional neighborhood of discriminant components and the elliptic fibration over it, $(\cdot, \cdot)$ happens to be the topological intersection product. Since all vanishing cycles $\pi_{i}$ are the same for $A_{r}$ it took a simple form; this is generically not the case. In this section we derive the generic form.

Consider a junction $J$ of the discussed type. The self intersection $(J, J)$ receives contributions from vanishing cycles and also from junction points; the latter involve two prongs becoming one in $B$ away from components of the discriminant, locally giving a pair of pants in $X$, as depicted in figure 2.1. Heuristically, we denote these two types of contributions a: 11

$$
(J, J)=(J, J)_{v}+(J, J)_{j} \equiv J_{k} I_{k l} J_{l}=J^{T} I J
$$

For junctions constructed from $N$ components $\Delta_{i}$, the symmetric matrix $I_{k l}$ gives a symmetric bilinear form in the junction basis. We write this matrix in terms of contributions from vanishing cycles and junction points as $I_{k l}=I_{k l}^{v}+I_{k l}^{j}$. The vanishing cycle contribution is straightforward: in a sufficiently small neighborhood of a puncture, $J$ intersects itself only at the puncture. For a particular $l$, if $J_{l}=1$ the contribution from this vanishing cycle is -1 . More generically it is $-J_{l}^{2}$. Therefore we have $I^{v}=-\mathbf{1}_{N \times N}$.

In order to determine the contribution $(J, J)_{j}$ from junction points it will be useful to study an example which easily generalizes. Consider a junction with four punctures, choose an ordering, and construct pairs of pants in a small neighborhood in an ascending fashion, as depicted in figure 2.1. Recall that a junction with coordinate $J_{i} \in \mathbb{Z}$ on $\pi_{i}$ wraps the one-cycle $J_{i} \pi_{i}$. At the junction point where $J_{1} \pi_{1}$ and $J_{2} \pi_{2}$ join, for example, there are $J_{1} \pi_{1} \cdot J_{2} \pi_{2}$ non-trivial intersections in the torus fiber due to the joining of three one-cycles. These points contribute to the self-intersection $(J, J)_{j}$. It would seem that this contribution depends on the choice of two legs $J_{1} \pi_{1}$ and $J_{2} \pi_{2}$ instead of the other leg $-J_{1} \pi_{1}-J_{2} \pi_{2}$ (negative sign so that all one-cycles are "incoming"), but this is not so since $J_{1} \pi_{1} \cdot J_{2} \pi_{2}=J_{2} \pi_{2} \cdot\left(-J_{1} \pi_{1}-J_{2} \pi_{2}\right)=$ $\left(-J_{1} \pi_{1}-J_{2} \pi_{2}\right) \cdot J_{1} \pi_{1}$. The contribution from all three junction points to the self-intersection is therefore given by $J_{1} \pi_{1} \cdot J_{2} \pi_{2}+\left(J_{1} \pi_{1}+J_{2} \pi_{2}\right) \cdot J_{3} \pi_{3}+\left(J_{1} \pi_{1}+J_{2} \pi_{2}+J_{3} \pi_{3}\right) \cdot J_{4} \pi_{4}=\sum_{i<j} J_{i} \pi_{i} \cdot J_{j} \pi_{j}$.

This argument generalizes directly to $n$-punctures; i.e., to $n$-pronged string junctions. At the $j$ th junction point, $\sum_{i=1}^{j} J_{i} \pi_{i}$ forms a pair of pants with $J_{j} \pi_{j}$. The contribution to the self-intersection from all of the points is given by

$$
(J, J)_{j}=\sum_{j=1}^{n-1}\left(\sum_{i=1}^{j-1} J_{i} \pi_{i}\right) \cdot J_{j} \pi_{j}=\sum_{i<j} J_{i} \pi_{i} \cdot J_{j} \pi_{j} \equiv J_{m} U_{m n} J_{n}
$$

where $U$ is the upper triangular $N \times N$ matrix with entries for $i<j$ given by $\pi_{i} \cdot \pi_{j}$. Defining a symmetric matrix $I^{j}=\frac{1}{2}\left(U+U^{T}\right)$ gives $(J, J)_{j}=J_{m} I_{m n}^{j} J_{n}$.

Computing the topological intersection matrix $I$ only requires knowing the ordered set $Z$ of vanishing one-cycles, which determines the junction basis. For any given geometry, there are many equivalent choices of $Z$, but the product (2.2) is invariant, as we will now show.

\footnotetext{
${ }^{11}$ While the transpose is explicit here, it will be implied elsewhere in the text, though hopefully clear based on context.
} 


\subsection{Picard-Lefschetz Theory}

In the last four sections we have asked the reader to suspend disbelief and take an ordered set of vanishing cycles $Z$ as input for an analysis of two-cycles in an elliptic surface. In this section we justify this input data and discuss a method for determining $Z$.

Before discussing its relevance for our work, let us review the basic ideas of Picard-Lefschetz theory. Consider a holomorphic map $f: Y \rightarrow B$ where $Y$ is a $d$ dimensional compact complex manifold and $B$ is $\mathbb{P}^{1}$. Let the patches of $B$ be the northern and southern hemispheres $P_{+}$and $P_{-}$, chosen so that all of the non-degenerate critical points $\left\{p_{1}, \ldots, p_{k}\right\}$ are in the interior of $P_{+}$, and let $z$ be a coordinate on $P_{+}$. Pick a base point $P$ and remove the singular points and associated singular fibers from $Y$ and $B$, defining $\tilde{Y}=Y \backslash f^{-1}\left\{p_{1}, \ldots, p_{k}\right\}$ and $\tilde{B} \equiv B \backslash\left\{p_{1}, \ldots, p_{k}\right\}$. Let $E_{P}$ be the smooth elliptic fiber at $P$.

The fundamental group $\pi_{1}(\tilde{B}, P)$ is non-trivial and induces an action on the homology of $Y$ according to the Picard-Lefschetz formula. Let us describe its generators. Choose a disk with center $p_{i}$ in the interior of $P_{+}$, and let the disk have radius $\rho>0$ small enough such that any $p_{j}$ with $i \neq j$ is outside of the disk. Let $\chi_{i}(s)=p_{i}+\rho e^{2 \pi i s}$ with $0 \leq s \leq 1$ be a circular path around $p_{i}$, and let $l_{i}$ be any path from $P$

to $p_{i}+\rho$ in $\tilde{B}$. Then $w_{i}=l_{i}^{-1} \circ \chi_{i} \circ l_{i}$ is called the elementary path encircling $p_{i}$. The fundamental group is generated by the homotopy classes $\left[w_{1}\right], \ldots,\left[w_{k}\right]$ of the elementary paths. Associated to each $p_{i}$ is a vanishing cycle $\pi_{i}$, which we will soon determine. Given these vanishing cycles, the Picard-Lefschetz theorem gives the action of the fundamental group on the homology. For $p \neq d-1$ the fundamental group acts trivially on $H_{p}(Y)$. For $p=d-1$ the elementary path $w_{i}$ acts on an element $x$ of $H_{p}(Y)$ as

$$
w_{i}(x)=x+(-1)^{d(d+1) / 2}\left\langle x, \pi_{i}\right\rangle \pi_{i} .
$$

This result is due to Picard for surfaces $(d=2)$, and to Lefschetz in the higher dimensional case. For a recent discussion of this effect in the language of $(p, q)$ seven-branes and $S L(2, \mathbb{Z})$ monodromy, see [60].

The relevance to our work is clear. Consider the elliptic fibration $X_{d}$ with discriminant $\Delta \sim z^{N}(\ldots)$. Define the patch $P_{+}$to be a disk with center $z=0$, and deform $X_{d}$ such that the $N$-fold degenerate critical point at $z=0$ becomes $N$ non-degenerate critical points at $\left\{p_{1}, \cdots, p_{N}\right\}$ in $P_{+}$. This is in the spirit of the work of Arnol'd [22] and Gusein-Zade [23]. We can deform such that $p_{i} \neq 0$, so we choose the base point $P$ to be $z=0$. Schematically, for $N=5$ the setup appears as

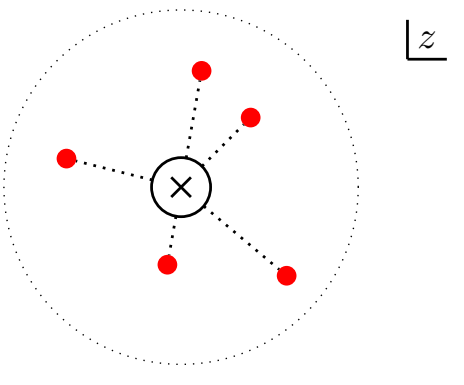

where the red dots are the $p_{i}$, the check at the origin is the base point $P$, the solid circle is a small neighborhood of $P$ and the large dotted circle is $P^{+}$. One can visualize the elementary paths $w_{i}$ going around each point $p_{i}$ by following the straight dotted line towards $p_{i}$, encircling it in a small neighborhood, and then following the dotted line back to the base point. When determining the vanishing cycles $\pi_{i}$, we 
will follow the dotted line all the way to the critical point, calling it a path of approach.

It is simple to determine an ordered set of vanishing cycles. Consider an elliptic fibration over $P^{+}$ given in Weierstrass form as

$$
y^{2}=x^{3}+f x+g
$$

where $f$ and $g$ are polynomials in $z$. At a generic point in the base the roots of the right hand side determine three marked points in the $x$-plane

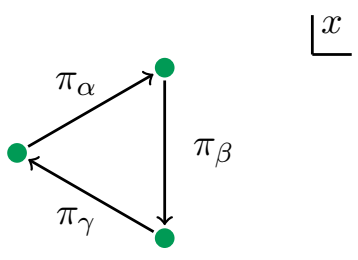

where we have drawn three paths $\pi_{\alpha}, \pi_{\beta}$, and $\pi_{\gamma}$ between them such that $\pi_{\alpha}+\pi_{\beta}+\pi_{\gamma}=0$. In fact, these paths are one-cycles: at the points where $x^{3}+f x+g=0$ the double cover (2.6) degenerates, but every other point on the path is a double cover, so that topologically the path is an $S^{1}$. More generic paths between the marked points also give one-cycles, and any two of the $\pi_{\alpha}, \pi_{\beta}$, and $\pi_{\gamma}$ can be taken as generators of $H_{1}\left(E_{P}, \mathbb{Z}\right)=\mathbb{Z}^{2}$.

The elliptic fiber becomes singular when $y=0, x^{3}+f x+g=0$, and $\frac{\partial}{\partial x}\left(x^{3}+f x+g\right)=0$. The latter two equations are satisfied if and only if the $x^{3}+f x+g$ has a double root, and thus at any $p_{i}$ two of the green points have collided. In following the path of approach from $P$ to $p_{i}$, those two green points take a particular path, and the homology cycle of this path is the vanishing cycle associated the point $p_{i}$. We will demonstrate this phenomenon for $A_{r}$ and $D_{4}$ cases in sections 3.2 and 5.2 , respectively. This data, together with the natural ordering of $p_{i}$ determined by the order of paths of approach around a neighborhood of $P$, determines the set $Z$ of ordered vanishing cycles which is the input for a junction analysis. For a $N$-element $Z$, we define the monodromy around the entire set $w_{Z}=w_{\pi_{N}} \circ \cdots \circ w_{\pi_{1}}$. We refer to the Picard-Lefschetz monodromy as the PL-monodromy, to distinguish it from the outer monodromy, which we have denoted by O-monodromy.

Let us study the effect of swapping the order of two adjacent points; to do so, it is sufficient to consider an example with three points $p_{i}$
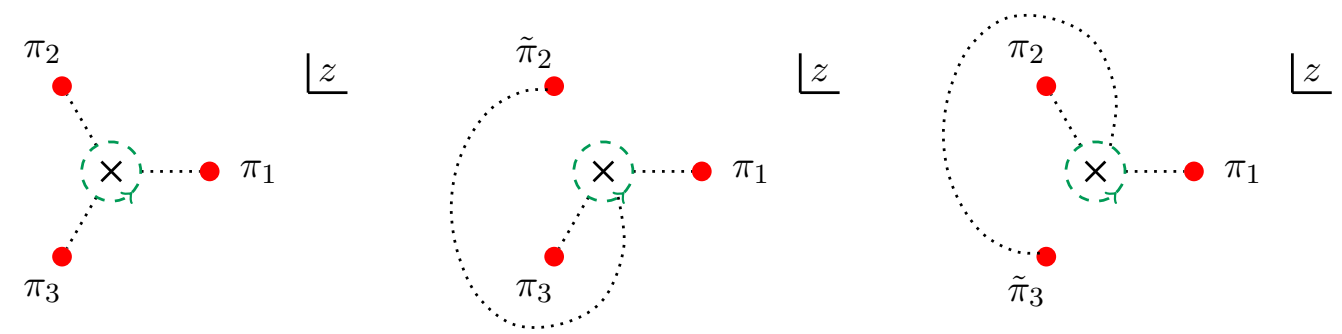

where the figure on the left-hand side corresponds to the set $Z_{l}=\left\{\pi_{1}, \pi_{2}, \pi_{3}\right\}$ by following the direction of the green arrow encircling the base point. The PL-monodromy on an arbitrary one-cycle $\gamma$ is $\left(w_{\pi_{3}} \circ w_{\pi_{2}} \circ\right.$ $\left.w_{\pi_{1}}\right)(\gamma)$. Suppose one chooses a path which goes around $p_{3}$ before $p_{2}$. There are two distinct choices, given by the center and right diagrams. Consider the center diagram. Since the path to $p_{3}$ has not changed, it must have the same vanishing cycle $\pi_{3}$, while the vanishing cycle associated to $p_{2}$ is now $\tilde{\pi}_{2}=w_{\pi_{3}}\left(\pi_{2}\right)=$ 
$\pi_{2}+\pi_{2} \cdot \pi_{3} \pi_{3}$ and $Z_{c}=\left\{\pi_{1}, \pi_{3}, \tilde{\pi}_{2}\right\}$. A simple computation shows that $w_{\tilde{\pi}_{2}}=w_{\pi_{3}} \circ w_{\pi_{2}} \circ w_{\pi_{3}}^{-1}$, and therefore $w_{Z_{c}}=w_{\tilde{\pi}_{2}} \circ w_{\pi_{3}} \circ w_{\pi_{1}}=w_{Z_{l}}$. A similar analysis with $\tilde{\pi}_{3}=w_{\pi_{2}}^{-1}\left(\pi_{3}\right)$ and $Z_{r}=\left\{\pi_{1}, \tilde{\pi}_{3}, \pi_{2}\right\}$ gives $w_{Z_{r}}=w_{Z_{l}}$. We see that the monodromy is invariant under changes of path in which two adjacent points in the path ordering are swapped.

Any change in path can be obtained by successive swaps, and therefore the PL-monodromy is invariant under such manipulations. Therefore, for a deformation of any ADE singularity the choice of an ordered set $Z$ is not unique, but instead all such $Z$ fall into an equivalence class with the same PL-monodromy; they are related by an elementary transformation of strong bases [23]. In particular, given a deformed ADE singularity the homology cannot depend on the choice of path which determines $Z$. Since $Z$ determines the junction basis this has important consequences for junctions: given two orderings $Z$ and $\tilde{Z}$ in the same equivalence class, the corresponding intersection matrices $I$ and $\tilde{I}$ will differ, as will the vectors in $\mathbb{Z}^{N}$ representing the junctions; however, they will have the same lattice structure. For example, we will see that certain one-parameter deformations of ADE singularities do not give rise to the canonical orderings $Z$ of Zwiebach and DeWolfe, but instead determine different sets $Z$. In both cases, though, junctions $J$ of with $(J, J)=-2$ and $a(J)=0$ give rise to the root lattice of the corresponding ADE algebra, despite giving different embeddings of the roots in $\mathbb{Z}^{N}$.

Let us comment on the product $(J, J)$. We would like to show that it is invariant under a swapping, and therefore under choice of path. Consider a junction $J=\left(J_{1}, J_{2}\right)$ with vanishing cycles $\left\{\pi_{1}, \pi_{2}\right\}$. From (2.2), we see that $(J, J)=-J_{1}^{2}-J_{2}^{2}+J_{1} J_{2} \pi_{1} \cdot \pi_{2}$. Performing the path swap so that the vanishing cycles are given by $\left\{\pi_{4}, \pi_{1}\right\}$ where $\pi_{4}=w_{\pi_{1}}^{-1}\left(\pi_{2}\right)$, the junction $J$ becomes $J=\left(J_{2}, J_{1}+J_{2} \pi_{2} \cdot \pi_{1}\right)$, and we compute

$$
\begin{aligned}
(J, J) & =-J_{2}^{2}-\left(J_{1}+J_{2} \pi_{2} \cdot \pi_{1}\right)^{2}+\left[J_{2}\left(\pi_{2}-\left(\pi_{2} \cdot \pi_{1}\right) \pi_{1}\right) \cdot\left(J_{1}+J_{2} \pi_{2} \cdot \pi_{1}\right)\right] \pi_{1} \\
& =-J_{1}^{2}-J_{2}^{2}+J_{1} J_{2} \pi_{1} \cdot \pi_{2}
\end{aligned}
$$

showing that the $(J, J)$ is invariant under the swap. A similar calculation shows that $(J, J)$ is invariant under the other path swap, where the paths give the ordering of vanishing cycles $\left\{\pi_{2}, \pi_{3}\right\}$ with $\pi_{3}=$ $w_{\pi_{2}}\left(\pi_{1}\right)$; the junction $J$ is $J=\left(J_{2}-J_{1} \pi_{1} \cdot \pi_{2}, J_{1}\right)$ in that basis.

Finally, many works on string junctions study the homological equivalence of junctions under so-called Hanany-Witten 24] moves. The basic idea is simple. Consider a junction $J$ with a junction point, as discussed above, and suppose that one of the prongs contributing to the junction point ends on a marked point with vanishing cycle $\pi_{i}$. The prong ending on it can disappear by moving the path $j_{1}$ in $B$ which determines the junction such that it crosses the marked point and is now a path $j_{2}$. In such a case the asymptotic charge is left invariant because the contribution from the lost prong is compensated for by the Picard-Lefschetz action of $\pi_{i}$ on the one-cycle above $j_{2}$. The two-cycles in $X$ determined by $j_{1}$ and $j_{2}$ are homologically equivalent. Without loss of generality, in this paper we study homological representatives of junctions with the maximal number of prongs; i.e. where there is no Picard-Lefschetz action on the onecycle above the corresponding path in $B$. This is natural for the deformations we consider and makes Lie algebraic computations considerably easier. See Figure 3 of [60] for a recent depiction of the equivalence of junctions under a Hanany-Witten move. 


\section{Deformations and ADE Algebras}

In this section we study surface deformations and ADE algebras using the techniques discussed in section 2. We begin by giving an ordered set $Z$ for each ADE algebra which allows for a junction analysis. We also perform a one-parameter smooth deformation of a singularity for each ADE algebra and determine an associated ordered set of vanishing cycles $Z$ which differs from the canonical examples in [15] for $D_{r}, E_{6}$, $E_{7}$, and $E_{8}$; nevertheless, the root lattices are isomorphic. We then discuss the appearance of non-trivial representations, a map from roots to weights in the Dynkin basis, and the importance and application of Freudenthal's recursion formula to junctions.

\subsection{The Canonical Basis}

In [15] particular sets $Z$ are used to study junctions filling out ADE representations. They are given by

$$
\begin{array}{ll}
A_{r}: & Z=\left\{\pi_{A_{1}}, \ldots, \pi_{A_{r+1}}\right\} \\
D_{r}: & Z=\left\{\pi_{A_{1}}, \ldots, \pi_{A_{r}}, \pi_{B}, \pi_{C}\right\} \\
E_{6}: & Z=\left\{\pi_{A_{1}}, \ldots, \pi_{A_{5}}, \pi_{B}, \pi_{C_{1}}, \pi_{C_{2}}\right\} \\
E_{7}: & Z=\left\{\pi_{A_{1}}, \ldots, \pi_{A_{6}}, \pi_{B}, \pi_{C_{1}}, \pi_{C_{2}}\right\} \\
E_{8}: & Z=\left\{\pi_{A_{1}}, \ldots, \pi_{A_{7}}, \pi_{B}, \pi_{C_{1}}, \pi_{C_{2}}\right\}
\end{array}
$$

where the subscripts on $A$ denote that there are multiple components with vanishing cycle $\pi_{A}$, and similarly for $C$. Note that the number of discriminant components $N$, and thus $\pi_{i}$ 's, satisfy $N=r+1$ for $A_{r}$ algebras and $N=r+2$ for the rest. Given this data, the monodromy can be easily computed using the PicardLefschetz formula (2.4). In this basis, the associated intersection products $I$ can be computed from the general formula (2.2) and are presented in Table 5. The results agree with [15]. We may refer to these canonical sets $Z$ in shorthand form as $A A A A B C$ or $A^{4} B C$ for $D_{4}$, for example.

\section{$3.2 \quad A_{r}$ Deformations}

We now turn to the deformation of $A_{r}$ algebras. The local equation of a Weierstrass model with $A_{r}$ surface singularities can be written as

$$
y^{2}=x^{3}-3 a^{2} x+2 a^{3}+z^{r+1} \quad \text { with } \quad a \neq 0 .
$$

We do not consider the most general deformation, but instead a one-parameter deformation which completely Higgses the gauge group. Such a deformation is given by

$$
y^{2}=x^{3}-3 a^{2} x+2 a^{3}+z^{r+1}-\varepsilon \quad \text { with } \quad \varepsilon \ll 1 .
$$


and the discriminant takes the form $\Delta=\left(z^{r+1}-\varepsilon\right)\left(2 a^{3}+z^{r+1}-\varepsilon\right)$. Since the deformed $A_{r}$ singularity arises entirely from the first factor, we study a neighborhood of $z^{r+1}-\varepsilon=0$, and the discriminant becomes

$$
\Delta \sim \prod_{j=1}^{r+1}\left(z-\varepsilon_{j}\right)
$$

where $\varepsilon_{j} \equiv|\varepsilon|^{1 /(r+1)} e^{2 \pi i j /(r+1)}$. For the $S U(4)$ case where $N=r+1=4$ the discriminant components have been deformed away from $z=0$ as

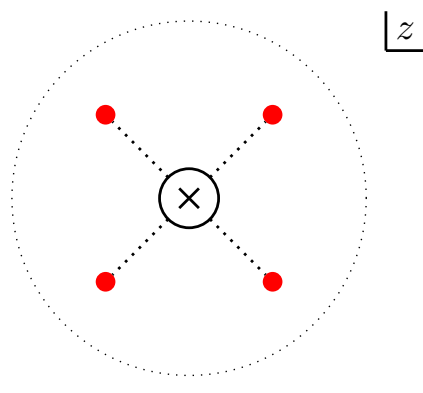

where an analogous result clearly applies to other values of $r$.

Let us determine the vanishing cycles, as described in section 2.5. Two of the roots of the right hand side of (3.3) collide at $z=\varepsilon_{j}$, making the elliptic fiber singular. It is clear that the same two roots must collide for any $z=\varepsilon_{j}$, since the phase data does not enter into (3.3) due to the $r+1$ power. Thus, each of the $r+1$ marked points have the same vanishing cycle, which we can take to be $\pi_{A}$ in a particular $S L(2, \mathbb{Z})$ frame, matching the known result (3.1). With this $Z$, one can proceed with a junction analysis. The results are as discussed in section 2.3 .

\section{3 $\quad D_{r}$ Deformations}

Let us proceed similarly in the $D_{r}$ case. We consider the local equation of a Weierstrass model with $D_{r}$ singularities along $z=0$ :

$$
y^{2}=x^{3}-3 c^{2} z^{2} x+2 c^{3} z^{3}+\mu z^{r-1}
$$

where $y$ and $x$ are again fiber coordinates and $c, \mu$ are parameters. This is always the case if $r \geq 5$. Consider a simple one-parameter deformation which completely Higgses the gauge group:

$$
y^{2}=x^{3}-3 c^{2} s^{2} x+2 c^{3} z^{3}+\mu z^{r-1}-\varepsilon .
$$

The discriminant is given by $\Delta=\left(z^{r-1}-\varepsilon\right)\left(4 c^{3} z^{3}+z^{r-1}-\varepsilon\right)$. Defining $a \equiv 4 c^{3}+z^{r-4}$ and studying $\Delta$ in a neighborhood of $z^{r-1}-\varepsilon=0$, we have

$$
\Delta \sim\left(z^{3}-\varepsilon / a\right) \prod_{j=1}^{r-1}\left(z-\varepsilon_{j}\right)
$$

where the $\varepsilon_{j}$ are again determined by $\varepsilon$. We see that the discriminant has split into $N=r+2$ components, which are unique for generic values of $a$. The $D_{r}$ gauge theory is completely Higgsed. In section 5.2 we 
explicitly determine the vanishing cycles in the $D_{4}$ case. The set $Z_{D_{4}}=\left\{\pi_{\alpha}, \pi_{\beta}, \pi_{\gamma}, \pi_{\alpha}, \pi_{\beta}, \pi_{\gamma}\right\}$ that we find is different from the set $Z=\left\{\pi_{A}, \pi_{A}, \pi_{A}, \pi_{A}, \pi_{B}, \pi_{C}\right\}$ of the canonical basis (3.1), but are equivalent under an elementary transformation of strong bases, as discussed in section 2.5. For $D_{5}$ we have derived the set is given by $Z_{D_{5}}=\left\{\pi_{\alpha}, \pi_{\alpha}, \pi_{\beta}, \pi_{\gamma}, \pi_{\alpha}, \pi_{\beta}, \pi_{\gamma}\right\}$. Though we have not derived the generic result directly from geometry, a natural guess given this pattern is $Z_{D_{4+k}}=\left\{\pi_{\alpha_{1}}, \ldots, \pi_{\alpha_{k}}, \pi_{\alpha}, \pi_{\beta}, \pi_{\gamma}, \pi_{\alpha}, \pi_{\beta}, \pi_{\gamma}\right\}$; this matches expectations from the canonical basis, and moreover we have explicitly checked the root junctions for this $Z_{D_{4+k}}$ up through the $D_{8}$ case, finding agreement.

\section{4 $E_{6}, E_{7}$, and $E_{8}$ Deformations}

In this section we give the surface deformations of $E_{6}, E_{7}$, and $E_{8}$.

Consider the deformed local Weierstrass model

$$
y^{2}=x^{3}+c z^{k}-3 \varepsilon(x+1)
$$

where the $E_{6}$ case has $k=4$ and the $E_{8}$ case has $k=5$. In the $\varepsilon \rightarrow 0$ limit the deformation is undone and the Weierstrass model has singularities along $z=0$. The discriminant is given by $\Delta=\left(c z^{k}-3 \varepsilon\right)^{2}-4 \varepsilon^{3}$ and we see that the discriminant splits into two sets of $k$ marked points, the solutions of $c z^{k}-3 \varepsilon= \pm 2 \varepsilon^{3 / 2}$. For generic values of the parameters the discriminant is non-degenerate and the gauge group is completely Higgsed. Performing an analysis as in section 2.5, we find that that for this deformation the ordered set of vanishing cycles are $Z_{E_{6}}=\left\{\pi_{\alpha}, \pi_{\gamma}, \pi_{\alpha}, \pi_{\gamma}, \pi_{\alpha}, \pi_{\gamma}, \pi_{\alpha}, \pi_{\gamma}\right\}$ and $Z_{E_{8}}=\left\{\pi_{\alpha}, \pi_{\gamma}, \pi_{\alpha}, \pi_{\gamma}, \pi_{\alpha}, \pi_{\gamma} \pi_{\alpha}, \pi_{\gamma}, \pi_{\alpha}, \pi_{\gamma}\right\}$. We see again that simple deformations of surface singularities do not reproduce the canonical junction basis (3.1). Taking specific values for the one-cycles, $\pi_{\alpha}=\pi_{A}$ and $\pi_{\gamma}=-\pi_{C}$, a junction analysis using $Z_{E_{6}}$ and $Z_{E_{8}}$ as input data finds 72 and 240 junctions $J$ with $(J, J)=-2$ and $a(J)=(0,0)$, matching

the number of roots of $E_{6}$ and $E_{8}$ as expected. Furthermore, in the $E_{6}$ case there are 27 junctions $J$ with $(J, J)=-1$ and $a(J)=(1,0)$, as expected from the canonical basis $A A A A A B C C$. The intersection matrix $I$ for $Z_{E_{6}}$ is given by

$$
I=\left(\begin{array}{cccccccc}
-1 & 1 / 2 & 0 & 1 / 2 & 0 & 1 / 2 & 0 & 1 / 2 \\
1 / 2 & -1 & -1 / 2 & 0 & -1 / 2 & 0 & -1 / 2 & 0 \\
0 & -1 / 2 & -1 & 1 / 2 & 0 & 1 / 2 & 0 & 1 / 2 \\
1 / 2 & 0 & 1 / 2 & -1 & -1 / 2 & 0 & -1 / 2 & 0 \\
0 & -1 / 2 & 0 & -1 / 2 & -1 & 1 / 2 & 0 & 1 / 2 \\
1 / 2 & 0 & 1 / 2 & 0 & 1 / 2 & -1 & -1 / 2 & 0 \\
0 & -1 / 2 & 0 & -1 / 2 & 0 & -1 / 2 & -1 & 1 / 2 \\
1 / 2 & 0 & 1 / 2 & 0 & 1 / 2 & 0 & 1 / 2 & -1
\end{array}\right)
$$

and the intersection matrix for $Z_{E_{8}}$ can also be computed easily. We leave an in-depth analysis of these sets $Z_{E_{6}}$ and $Z_{E_{8}}$ for future work.

Let us turn to the $E_{7}$ case. Consider the deformed Weierstrass equation

$$
y^{2}=x^{3}+\left(3 c z^{3}-3 \varepsilon\right) x+2 \varepsilon
$$


which has an $E_{7}$ singularity along $z=0$ in the $\varepsilon \rightarrow 0$ limit. The discriminant is given by $\Delta=\left(c z^{3}-\right.$ $\varepsilon)^{3}+\varepsilon^{2}$ and components $\Delta_{i}$ break into three sets of 3 marked points, the solutions of $c z^{3}-\varepsilon=l$ with $l$ a third root of $-\varepsilon^{2}$. The ordered set of vanishing cycles associated with this deformation is $Z_{E_{7}}=$ $\left\{\pi_{\alpha}, \pi_{\beta}, \pi_{\gamma}, \pi_{\alpha}, \pi_{\beta}, \pi_{\gamma}, \pi_{\alpha}, \pi_{\beta}, \pi_{\gamma}\right\}$ and performing a junction analysis with the concrete values $\pi_{\alpha}=\pi_{A}$,

$\pi_{\beta}=\left(\begin{array}{c}0 \\ 1\end{array}\right), \pi_{\gamma}=-\pi_{C}$, one discovers there are 126 junctions $J$ with $(J, J)=-2$ and $a(J)=0$. This matches the number of roots of $E_{7}$, as expected. We leave an in-depth study of this $Z_{E_{7}}$ for future work.

\subsection{Non-trivial Representations and Freudenthal's Formula}

Having discussed deformations of ADE singularities and the realization of ADE root systems as $r$ dimensional lattices in $\mathbb{Z}^{N}$, we will now utilize this formalism to describe non-trivial representations.

The representation theory of Lie algebras is rich. Let us briefly remind the reader of the basic formalism. A non-trivial representation $\rho$ of a simple Lie algebra $\mathfrak{g}$ is determined by a highest weight vector $\rho$, clearly abusing notation. Any weight in the weight lattice of $\rho$ can be obtained by subtracting some number of simple roots $\alpha_{i}$ from $\rho$. For example, the weight lattice of the adjoint representation $\operatorname{ad}(\mathfrak{g})$ is the root lattice, and all roots can be obtained by subtracting simple roots from the highest root. The algorithm of the previous section 12 of searching for junctions $J$ with $(J, J)=-2$ and $a(J)=\left(\begin{array}{l}0 \\ 0\end{array}\right)$ recovered the root lattices of the ADE algebras precisely because they are simply-laced; that is, all non-trivial roots $J$ of ADE algebras have the same length $(J, J)=-2$ and multiplicity one. A generic representation does not satisfy this property, and therefore the previous algorithm is not applicable in general. In fact, even in the case of root lattices, the roots of the Cartan subalgebra were added by hand, knowing that they are trivial weights with multiplicity $r k(\mathfrak{g})$.

It is important to note that a set of junctions associated to a representation $\rho$ of $\mathfrak{g}$ are not the weights of $\mathfrak{g}$, since the rank of their span is typically greater than $r k(\mathfrak{g})$; rather, there is a map from junctions to weights. The former can be seen in a simple example. Consider $S U(2)$ : the 2 is composed of two junctions $J_{1}=(1,0)$ and $J_{2}=(0,1)$, which are those $J$ with $(J, J)=-1$ and $a(J)=\left(\begin{array}{c}1 \\ 0\end{array}\right)$. Clearly $r k\left(\operatorname{span}_{\mathbb{Z}}\left(J_{1}, J_{2}\right)\right)>r k(\mathfrak{g})$, and therefore it isn't technically correct to call $\left\{J_{1}, J_{2}\right\}$ the weight lattice of the 2. This is an artifact of embedding in the higher dimensional space $\mathbb{Z}^{N}$. Specifically, any vector in $\operatorname{span}_{\mathbb{Z}}\left(J_{1}, J_{2}\right)$ proportional to $J_{1}+J_{2}$ doesn't intersect any of the simple roots, and therefore can't be in the weight lattice. Taking into account this fact, the rank is correct. In general there will be $N-r$ such relations, since the roots span an $r$-dimensional subspace of $\mathbb{Z}^{N}$; these relations are derived by computing $\operatorname{ker}(R)$, where $R$ is the $N \times r$ matrix whose columns are the simple roots junctions.

It is simple to give a generic map from junctions to weights; for simplicity, we will choose to use the Dynkin basis, where the simple roots are vectors given by the rows of the Cartan matrix. A map $F$ from a set of junctions $J$ to the weight lattice in the Dynkin basis is a map $F: \mathbb{Z}^{N} \rightarrow \mathbb{Z}^{r}$, and this can be computed easily. Since the intersection of root junctions gives the negative Cartan matrix $-A$, we have $R^{T} I R=-A$, where $I$ is the intersection product (2.2). Since the columns of $R$ are the root junctions in $\mathbb{Z}^{N}$, we see that $F=-R^{T} I$ maps the roots to the Cartan matrix, and therefore their Dynkin labels. In the canonical basis (3.1) the maps $F$ from junctions to Dynkin labels match the results of [15]. They are

\footnotetext{
${ }^{12}$ This algorithm was advocated for in [15] and used to great effect, when applicable.
} 
in Table 6 for convenience. Since this map exists we will often abuse language and refer to junctions and weights interchangeably.

Much of the junction literature thus far has focused on simple representations. In a generic representation, the weights $\lambda$ have a variety of lengths and the multiplicities are non-trivial. The multiplicity $m_{\lambda}$ of arbitrary weight $\lambda$ can be determined by Freudenthal's formula

$$
\left[(\rho+\xi)^{2}-(\lambda+\xi)^{2}\right] m_{\lambda}=2 \sum_{\alpha>0} \sum_{j \geq 1} m_{\lambda+j \alpha}
$$

where $\rho$ is the highest weight and $\xi$ is the Weyl vector $\xi \equiv \frac{1}{2} \sum_{\alpha>0} \alpha$, given by half the sum of the positive roots. Since $m_{\rho}=1$, the multiplicities of all weights in a representation can be determined recursively.

If deformation theory and string junctions are to reproduce the generic structure of ADE algebras, junctions must be able to describe arbitrary representations. For non-trivial representations with weights of a variety of lengths and multiplicities, this this amounts to the question of whether Freudenthal's formula holds for junctions. The formula (3.12) requires a product on the algebra, which for junctions is given by (2.2), and the weight junctions are vectors in $\mathbb{Z}^{N}$, not $\mathbb{Z}^{r}$, as emphasized previously. It is simple to check that the highest root junctions reproduce the root lattices in $\mathbb{Z}^{N}$, as they should, but in lieu of a mathematical proof it is also important to check non-trivial examples.

We have performed many non-trivial checks in carrying out the computations in this paper, but let us briefly discuss one that may convince the reader the Freudenthal's formula can be utilized for junctions. Consider the case of $\mathfrak{g}=E_{6}$. In the Dynkin basis, the highest weight of the adjoint representation is $(0,0,0,0,0,1)$ and, as mentioned, Freudenthal's recursion formula reproduces the correct root lattice from this data. With the simple roots as given in Table 2, the highest root is given by $J_{h r}=(1,1,1,1,0,-2,-1,-1) . \quad E_{6}$ also has a representation 13 with highest weight $(0,0,0,0,0,3)$ in the Dynkin basis, and this representation has dimension 43,758. If Freudenthal's formula applies to junctions, it must compute a representation of dimension 43,758 when applied to a highest weight junction of $J_{b i g} \equiv 3 J_{h r}$; indeed, it does14. Similar methods will be utilized elsewhere in the paper, including discussions of the 126 dimensional representation of $S O(10)$ often considered in models of particle physics.

\section{Massless Matter in Codimension Two}

Having discussed the basic formalism in section 2 and the deformation of ADE singularities and associated representation theoretic data in section 3, we will now turn to discuss massless matter representations.

As we have seen, non-trivial representations of a Lie algebra $\mathfrak{g}$ can be identified with two-manifolds which emanate from the deformation of a codimension one ADE singularity. Vector multiplets in the adjoint representation arise as two-spheres $J$ with $(J, J)=-2$; as they do not have boundary, the asymptotic charge is $a(J)=(0,0)$. Other representations $\rho$ arise from junctions which wrap a non-vanishing one-cycle $a(J) \neq(0,0)$ a finite distance from $z=0$, and thus appear to have a boundary $b$ in this neighborhood. However, it is possible that $b$ ends on other seven-brane components $\Delta_{o}$, giving a two-cycle which may become massless on the codimension two locus $\Delta_{z} \cap \Delta_{o}$. Since this requires that the boundary $b$ "pinch

\footnotetext{
${ }^{13}$ See, e.g. table 47 of [87] for a list of irreducible $E_{6}$ representations of dimension $<100,000$.
}

${ }^{14}$ The interested reader may consult the computation in the code referenced in appendix B. 
off", the asymptotic charge $a(J)$ puts constraints on the allowed $\Delta_{o}$. We would like to again stress a main point: the data of a representation $\rho$ of $\mathfrak{g}$ arises from codimension one data of the elliptic fibration; the importance of codimension two data is that it determines whether or not the two-cycles associated to $\rho$ shrink to zero size on that locus, giving rise to massless matter.

There are many works $[9-14,26]$ studying the codimension two localization of matter via singularity resolution. These works have employed a number of approaches, and in this section we discuss three of them from the point of view of deformations. Specifically, we discuss codimension two singularities arising in $K 3$-fibrations, where representations $\rho$ of $\mathfrak{g}$ arise from the branching of adjoints of an enhanced codimension two algebra $\mathfrak{g}^{\prime}$, as utilized in [9]. We study the case of codimension two singularities with simple normal crossing, and argue that ideas from $K 3$-fibrations can be applied in this context as well. We also discuss outer automorphisms of $\mathfrak{g}$ induced by O-monodromy around codimension two loci; this phenomenon can break $\mathfrak{g}$ to a non-simply laced group $\mathfrak{g}^{\prime}$ and give non-trivial representations of $\mathfrak{g}^{\prime}$ in codimension two. We will exhibit all of these ideas in concrete junction examples in section 5. Due to the many possibilities, we do not attempt to present an exhaustive list of codimension two enhancements and their study via deformation. For further examples in the resolution picture, see [13] or [14].

We emphasize that in all three approaches the appearance of massless matter in codimension two can be understood in terms of junctions in families of elliptic surfaces. In these cases the Lie algebra product $(\cdot, \cdot)$ is in fact the topological intersection of two-cycles, represented as junctions.

\section{Elliptic K3-fibrations and Heterotic Duality}

Consider the case where $X$ is not only elliptically fibered, but also admits an elliptic $K 3$ fibration $X_{d} \stackrel{\pi_{K}}{\longrightarrow} B_{d-2}$ where the K3 fiber is in the stable degeneration limit. In this case the elliptic $K 3$ splits into two rational elliptic surfaces meeting along a common elliptic curve. We denote such $X$ as $\tilde{X}$ for convenience. Compactifications of F-theory on $\tilde{X}$ admit heterotic duals [2, 18, 19, where the heterotic compactification manifold $Y$ is an elliptically fibered Calabi-Yau $(d-1)$-fold over $B_{d-2}$. Indeed, via an appropriate specialization in the complex structure moduli space of $\tilde{X}$, an $E_{8}$ gauge theory can be engineered at codimension one in each of the rational elliptic surfaces.

Heterotic duality necessitates the existence of the string junction picture. This can be seen as follows. The heterotic dual is endowed with a holomorphic vector bundle $V_{1} \oplus V_{2}$ on $Y$. The bundle $V_{1}$ with structure group $H$ breaks one $E_{8}$ factor to a group $G=\left[E_{8}, H\right]$. If $V_{1}$ is trivial the entire $E_{8}$ gauge theory is intact and therefore the $240 \mathrm{~W}$-bosons of $E_{8}$ are massless; turning on bundle moduli such that the structure group $H=E_{8}$, the gauge group is completely broken and all $240 \mathrm{~W}$-bosons receive a mass. The F-theory dual of this process is the complete deformation of the $E_{8}$ singularity in one of the rational elliptic surfaces, and therefore 240 finite volume two-cycles must arise from deformation. We emphasize that these are not the resolution two-cycles, but instead are junctions represented as vectors in $\mathbb{Z}^{10}$.

Let us give an explicit description of the (Calabi-Yau) geometry $\tilde{X}$ before discussing the appearance of matter in codimension two. It is defined by the Weierstrass equation $y^{2}=x^{3}+f x+g$ with $f \in \Gamma\left(K_{B_{d-1}}^{-4}\right)$ and $g \in \Gamma\left(K_{B_{d-1}}^{-6}\right)$ as usual, but since $\tilde{X}$ is fibered by elliptic $K 3$ the base $B_{d-1}$ is itself $\mathbb{P}^{1}$ fibered over $B_{d-2}$, so that

$$
f=\sum_{a=0}^{8} z^{a} s^{8-a} f_{a} \quad g=\sum_{b=0}^{12} z^{a} s^{12-b} g_{b}
$$


where $f_{a} \in H^{0}\left(B_{d-2}, K_{B_{d-2}}^{-4} \otimes \mathcal{O}_{B_{f}}(\tilde{\eta})^{\otimes(4-a)}\right)$ and $g_{b} \in H^{0}\left(B_{d-2}, K_{B_{d-2}}^{-6} \otimes \mathcal{O}_{B_{d-2}}(\tilde{\eta})^{\otimes(6-b)}\right)$ are global sections dependent upon the choice 15 of a divisor class $\tilde{\eta}$ in $B_{d-2}$. Via the appropriate tuning of complex structure moduli in $f_{a}$ and $g_{b}$, an ADE singularity with group $G$ can be engineered along the component $\Delta_{z} \equiv\{z=0\}$ with multiplicity $N$. Suppose that there is another component of the discriminant $\Delta_{t}$ defined in terms of a local coordinate by $t=0$ which intersects $\Delta_{z}$ in codimension two, and that the ADE singularity enhances to $G^{\prime}$ at $\Delta_{z} \cap \Delta_{t}$ with multiplicity $N^{\prime}$.

In this case it is simple to see how matter arises. Consider $\tilde{X}$ in a neighborhood of $t=0$. This neighborhood specifies a family of elliptic $K 3$ 's $F_{t}$, and a generic $F_{t}$ has $N$ marked points at $z=0$ and $N^{\prime}-N$ marked points away from $z=0$. There exists a deformation of the $N$ marked points in a generic $F_{t}$ which gives junctions in the adjoint of $\mathfrak{g}$; they become massless upon undoing the deformation. Similarly, the $N^{\prime}$ marked points in the codimension two fiber $F_{0}$ yield an adjoint of $\mathfrak{g}^{\prime}$. In passing from $F_{0}$ to generic $F_{t}$ the $\operatorname{dim}(\mathfrak{g})-r k(\mathfrak{g})$ junctions between the $N$ marked points remain massless, giving a massless adjoint of $\mathfrak{g}$, while the other $\left(\operatorname{dim}\left(\mathfrak{g}^{\prime}\right)-r k\left(\mathfrak{g}^{\prime}\right)\right)-(\operatorname{dim}(\mathfrak{g})-r k(\mathfrak{g}))$ states become massive; the latter are the weights in $\operatorname{ad}\left(\mathfrak{g}^{\prime}\right)$ which aren't in $\operatorname{ad}(\mathfrak{g})$, and hence can be studied via branching rules $\operatorname{ad}\left(\mathfrak{g}^{\prime}\right)=\operatorname{ad}(\mathfrak{g}) \oplus_{i} \rho_{i}$. Running the process in reverse, junctions in the representations $\rho_{i}$ must become massless in codimension two.

\section{Simple Normal Crossing}

We have seen that under certain circumstances one can understand the appearance of matter in codimension two in terms of a family of elliptic K3's. In this section we would like to use a similar idea in the case of simple normal crossing. Here the discriminant takes the form $\Delta \sim z^{N} t^{N^{\prime}}$, where $z, t$ are local coordinates on $\mathbb{C}^{2}$. If one considers the elliptic fibrations over a one-dimensional neighborhood of $z=0$ in order to study the $N$ components associated to the algebra $\mathfrak{g}$, the other $N^{\prime}$ components do not give marked points as they did in the previous section. This is ultimately an artifact of the choice of neighborhood: if one considers the elliptic fibration over an appropriate slice

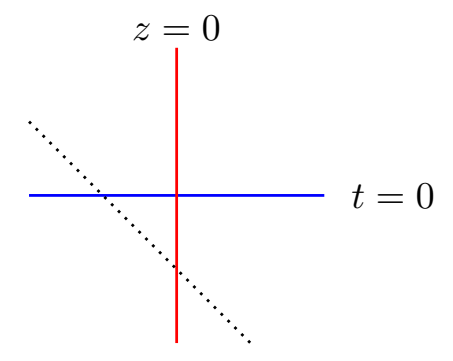

represented here in dots, the $N+N^{\prime}$ components all appear as marked points in this slice. This coordinate change allows one to study the algebra $\mathfrak{g}^{\prime}$ via junctions. The location of the $N^{\prime}$ marked points in relation to the $N$ marked points depends on $t$, and the techniques of the previous section can be applied in the same fashion.

A schematic picture may help the reader to visualize the process. For an $A_{3}$ enhancement to $A_{4}$, the

\footnotetext{
${ }^{15}$ The interested reader can find more details on heterotic F-theory duality, using identical notation, in 60].
} 
intersection of the discriminant with the the dotted slice is
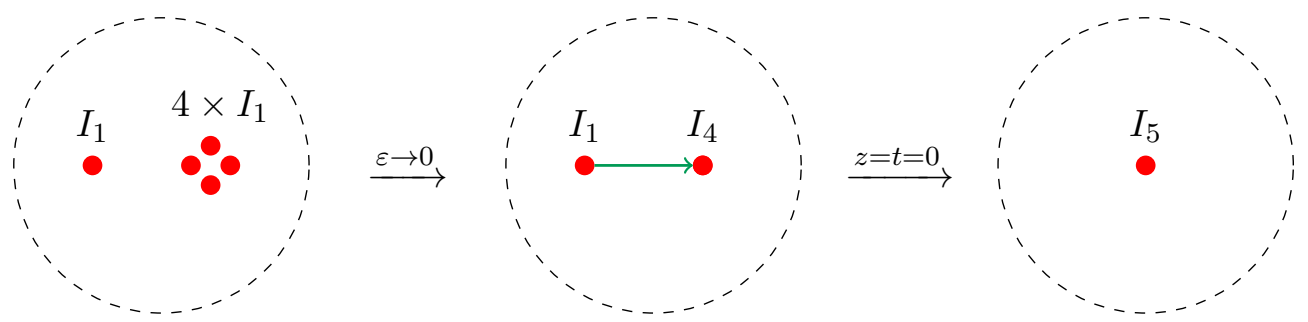

where on the left we have $\varepsilon \neq 0$, allowing for a junction analysis of the $A_{4}$ algebra which has been Higgsed; in the middle we have the $\varepsilon=0$ limit where the $S U(4)$ gauge symmetry is restored; and on the right we have the codimension two locus where the $I_{1}$ and $I_{4}$ singularity have collided, enhancing to $I_{5}$. The green arrow represents the motion of the $I_{1}$ and $I_{4}$ singularity towards one another as the plane moves closer towards $z=t=0$. As this happens, junctions stretching form the $I_{4}$ point to the $I_{1}$ point shrink to zero size, giving a massless fundamental and an antifundamental of $S U(4)$ there.

\section{O-Monodromy and Non-Simply-Laced Algebras}

Consider the locus $\Delta_{z}$. The generic fiber above this locus is singular with algebra $\mathfrak{g}$. The resolution of singularities gives rise to rational curves representing the extended Dynkin diagram of $\mathfrak{g}$, and for some elliptic fibrations these rational curves can be mapped to one another by taking a closed path around a codimension two locus $t=0$. This "O-monodromy" induces an outer automorphism on $\mathfrak{g}$. Since some nodes of the Dynkin diagram, and therefore $M 2$-brane states wrapped on them, cannot be distinguished under the monodromy, one must take the quotient of $\mathfrak{g}$ by the automorphism group, reducing $\mathfrak{g}$ to some non-simply-laced algebra $\mathfrak{g}^{\prime}$. This phenomenon is well-known in F-theory [10].

A natural question is whether a similar phenomenon holds in the deformation picture. If so, the Omonodromy action must act on two-cycles associated with deformation; i.e. on string junctions. It is simple to imagine how this might occur: considering a family of elliptic surfaces parameterized by codimension two data as discussed above, movement around a codimension two $t=0$ locus could permute deformed discriminant components and / or the one-cycles vanishing above them, inducing an action on the junction lattice. See section 5.2 for an explicit example of this phenomenon.

\section{$5 \quad$ Illustrative Examples}

In this section we study explicit examples which demonstrate the localization of massless matter representations in codimension two. We demonstrate the localization of matter in the deformation picture arising at a codimension two enhancement $A_{r} \rightarrow A_{r+1}$. We describe antisymmetrics of $S U(4)$ and sixteen dimensional spinors of $S O(10)$ via branching rules from higher rank algebras. We perform an explicit analysis of O-monodromy describing the reduction of $D_{4}$ to $G_{2}$. We also comment on a number of representations which are studied in models of particle physics.

To build intuition, we will not only study examples where the results are obtained directly from deformation, but will also study examples which use the canonical basis (3.1). The latter sometimes has the advantage that it is clear how the breaking of groups occurs, or coincides with IIb intuition. 


\subsection{Matter from Branching Rules}

In section 4 we described how massless codimension two matter can be understood by studying junctions in families of elliptic fibrations over a patch which depend on a parameter $t$. At $t=0$ a fiber collision enhances the algebra from $\mathfrak{g}$ to $\mathfrak{g}^{\prime}$ and the representations of $\mathfrak{g}$ at $z=t=0$ can be determined from branching rules. We demonstrate this explicitly in examples.

\section{Rank Enhancement}

Before proceeding on to more complicated examples, it is worthwhile to discuss a simple example. Consider the Weierstrass equation

$$
y^{2}=x^{3}-3 a^{2} x+2 a^{3}+\left(z^{r+1}-\varepsilon\right) t .
$$

In the $\varepsilon \rightarrow 0$ limit there is an $A_{r}$ singularity along $z=0$ which enhances to $A_{r+1}$ at $z=t=0$. The discriminant is given by $\Delta=\left(z^{r+1}-\varepsilon\right) t\left(2 a^{3}+\left(z^{r+1}-\varepsilon\right) t\right)$; near $t=0, z^{r+1}=\varepsilon$ we have

$$
\Delta \sim \prod_{j}^{r+1}\left(z-\varepsilon_{j}\right) t:=\Delta_{t} \prod_{j} \Delta_{z_{j}}
$$

and the simple normal crossing of each of the $r+1 \Delta_{z_{j}}$ components with the $\Delta_{t}$ component is evident. In the canonical junction basis the $A_{r}$ singularity is described by $r+1 A$-branes and at $t=0$ this stack collides with another $A$-brane, enhancing the algebra to $A_{r+1}$. Consider the simple example $r=1$, where $\mathfrak{g}=S U(2)$ and $\mathfrak{g}^{\prime}=S U(3)$. The roots of $\mathfrak{g}^{\prime}$ are given by

$$
\begin{array}{llll}
v_{1}=(1,0,-1) & v_{2}=(1,-1,0) & v_{3}=(0,1,-1) & v_{4}=(0,0,0) \\
v_{5}=(0,0,0) & v_{6}=(0,-1,1) & v_{7}=(-1,1,0) & v_{8}=(-1,0,1) .
\end{array}
$$

Upon moving from $F_{0}$ to $F_{t}, N^{\prime}-N=1$ of the A-branes moves away from $z=0$; choose it to be the first in the basis for convenience. Then $v_{3}, v_{4}$, and $v_{6}$ still give massless two cycles in the adjoint of $S U(2)$, and the extra Cartan element $v_{5}$ of $S U(3)$ is a singlet of $S U(2)$. The pairs $\left\{v_{1}, v_{8}\right\}$ and $\left\{v_{2}, v_{7}\right\}$ fill out a 2 of $S U(2)$ which is massive away from $t=0$ due to the separation between the $N$ marked point of the $S U(2)$ algebra and the extra marked point of the Higgsed $S U(3)$ algebra. These multiplets become massless upon taking $t \rightarrow 0$, matching the well known result that fundamentals of $S U(2)$ are localized at codimension two points of $S U(2) \rightarrow S U(3)$ enhancement. This same type of analysis holds for the generic $A_{r} \rightarrow A_{r+1}$ enhancement given above.

Let us consider examples purely using intuition from junctions. Consider a geometry which realizes an $A_{3}$ singularity along $z=0$, but enhances to $D_{4}$ at $z=t=0$. In the canonical junction basis the $A_{3}$ is represented 16 by $A A A A$ and $D_{4}$ is represented by $A A A A B C$, and thus $A A A A$ collides with $B C$ in codimension two. The adjoint representation of $D_{4}$ has dimension 28. The associated roots are presented in table 3, and the coloring in the table shows how the the adjoint of $D_{4}$ branches into representations of $A_{3}$. For example, the highest root of $D_{4}$ is $(1,1,0,0,-1,-1)$, and studying the representation of $A_{3}$

\footnotetext{
${ }^{16}$ In the weakly coupled type IIb limit this geometry describes the intersection of 4 D7-branes and an O7-plane, which can be obtained by unfolding a stack of D7-branes with $S O(8)$ gauge symmetry.
} 
requires dropping the last two entries of the junction, since they don't end on the first four entries which give the $A A A A$ of $A_{3}$ near a common codimension one locus. Doing so, we see the junction $(1,1,0,0)$ which has asymptotic charge $(2,0)$; studying this representation using Freudenthal's formula and the $A_{3}$ algebra shows that it is the 6 of $S U(4)$. Another simple example of branching is given in table 4. There we consider a $D_{5}$ enhancement to $E_{6}$ in codimension two, represented in the canonical junction basis by $A A A A A B C \rightarrow A A A A A B C C$, i.e. a $C$-brane collides with the $D_{5}$ singularity in codimension two. The highest root of $E_{6}$ is $(1,1,1,1,0,-2,-1,-1)$; forgetting the last coordinate in order to study junctions in representations of $D_{5}$ gives $(1,1,1,1,0,-2,-1)$, which is the highest weight of a $\mathbf{1 6}$ of $\mathrm{SO}(10)$.

\subsection{O-monodromy and Reducing $D_{4}$ to $G_{2}$}

In this section we study an example where the generic fiber in $\Delta_{z}$ exhibits a $D_{4}$ singularity, but monodromy around the codimension two locus $z=t=0$ induces an outer automorphism on the $D_{4}$ Dynkin diagram which reduces the gauge group to $G_{2}$. While the full automorphism group of $D_{4}$ is $S_{3}$, it is easy to see

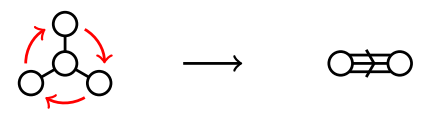

that the quotient of $D_{4}$ by the $Z_{3}$ subgroup of $S_{3}$ yields $G_{2}$ at the level of Dynkin diagrams and Lie algebras. This is realized geometrically by the smooth resolution of the local Weierstrass equation

$$
y^{2}=x^{3}-3 c^{2} z^{2} x+2 c^{3} z^{3}+a t z^{3}
$$

with its outer monodromy in $H_{2}(K, \mathbb{Z})$, where $K$ is a general surface intersecting the discriminant locus $z=0$. In $B$ there is a simple normal crossing intersection of $z=0$ and $t=0$; the general fiber over $z=0$ is $I_{0}^{*}$ while over a general point of $t=0$ it is $I_{1}$. The intersection of the discriminant with the $\operatorname{Re}(t)-\operatorname{Re}(z)$ plane is

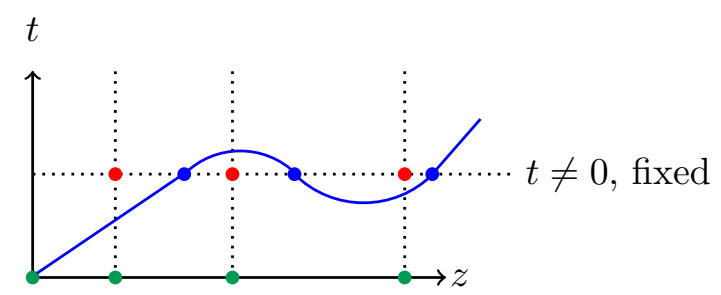

where the blue dots come $t \neq 0$ fixed coalesce at $t=0$ while the red dots remain fixed for all $t$.

The question we address in this section is whether the appearance of $G_{2}$ can be seen in the deformation picture. If it is possible, the monodromy around $t=0$ must induce an action on the six vanishing cycles associated with $D_{4}$, and the induced action on the string junctions representing simple roots must permute the outer legs of the Dynkin diagram while keeping the central node fixed. The relevant local Weierstrass equation, after deformation, is given by

$$
y^{2}=x^{3}-3 c^{2} z^{2} x+2 c^{3} z^{3}+a t z^{3}+\varepsilon t
$$


where $x$ and $y$ are local coordinates of the ambient space containing the elliptic fiber, $c$ and $a$ are parameters of the elliptic fibration, $z$ and $t$ are local coordinates in $B$ and $\varepsilon$ is a deformation parameter. We denote the fiber at a given $z$ and $t$ by $E_{z, t}$. The discriminant is given by

$$
\Delta=\left[\left(a t+4 c^{3}\right) z^{3}+\varepsilon t\right]\left(a z^{3}+\varepsilon\right) t .
$$

For a given $t \neq 0$, there are two sets of three singular points in the $z$-plane. Three are located at $a z^{3}+\varepsilon=0$ and the corresponding critical points in the Weierstrass model are $x=c z, y=0$; the other three occur at $\left(4 c^{3}+a t\right) z^{3}+\varepsilon t=0$ with corresponding critical points $x=-c z$, and $y=0$. In the $z$-plane for a given $t \neq 0$, let the former be denoted by red dots and the latter by blue dots. For appropriat 17 choices of $a$, $c$ (e.g. for $a, c$ real, $a<0, c>0$ ) and fixed real $t>0$ small enough, the marked points appear in the $z$-plane as

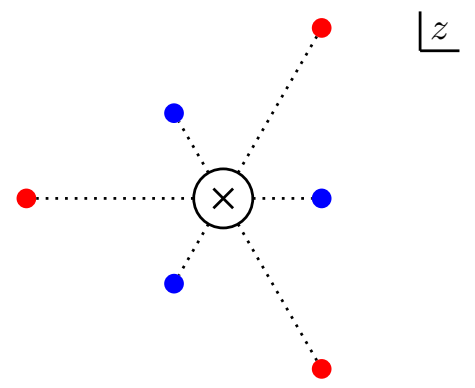

$\lfloor x$

where we choose the cross point at $z=0$ to be the base point $P$ and the solid circle near $P$ denotes the base point neighborhood discussed in section 2.5. The right-hand side gives a depiction of the complex $x$ line at $P$; the green marked points are solutions of $x^{3}+t \varepsilon=0$. The smooth fiber $E_{0, t}$ is given by the equation $y^{2}=x^{3}+t \varepsilon$, a branched cover of the complex $x$ line branched at the three green points (together with infinity). Consider a path in $x$ which meets roots only at the endpoints of the path; the inverse image in $E_{0, t}$ determines a closed (real) curve. For example the indicated paths give elements $\pi_{\alpha}, \pi_{\beta}$ and $\pi_{\gamma}$ in the first homology $H_{1}\left(E_{0, t} ; \mathbb{Z}\right)$; they satisfy the equation $\pi_{\alpha}+\pi_{\beta}+\pi_{\gamma}=0$ and any two of them form a basis. We can fix coordinates in $H_{1}\left(E_{0, t} ; \mathbb{Z}\right)$ so that $\pi_{\alpha}=\left(\begin{array}{l}1 \\ 0\end{array}\right), \pi_{\beta}=\left(\begin{array}{l}0 \\ 1\end{array}\right)$ and $\pi_{\gamma}=\left(\begin{array}{l}-1 \\ -1\end{array}\right)$. We also have, with the suitable (complex) orientation, $\pi_{\alpha} \cdot \pi_{\beta}=\pi_{\beta} \cdot \pi_{\gamma}=\pi_{\gamma} \cdot \pi_{\alpha}=1$.

Now suppose that we vary $z$ - holding $t$ fixed - along some path from zero to one of the six singular points in the $z$-plane without crossing any other of these points. As this happens, the roots of the equation in $x$

$$
x^{3}-3 c^{2} z^{2} x+2 c^{3} z^{3}+a t z^{3}+\varepsilon t=0
$$

will move from the green marked points until, in the singular point, two of them merge. This determines a path between these two points. The corresponding loop in $E_{0, t}$ shrinks to a point as $z$ moves along this path from the origin to the singular point, and the homology class is the associated vanishing cycle. For example, approaching the uppermost red point along the dotted line from $z=0$, the two roots on the edge labeled $\pi_{\alpha}$ coalesce. Furthermore, the path by which they join is homotopic relative endpoint:18, via paths that also can only touch green points at their endpoints, to the indicated path in the $x$-plane joining these two points; therefore at this point the cycle $\pi_{\alpha}$ vanishes. Similarly, moving counterclockwise

\footnotetext{
${ }^{17} \mathrm{~A}$ Mathematica notebook which demonstrates these phenomena is included in the supplementary files.

${ }^{18}$ i.e. the endpoints do not move through the homotopy.
} 
around the diagram beginning with the upper right red point, one can compute the rest of the vanishing cycles using straight line paths from the origin to the critical points. This determines the ordered set of vanishing cycles to be $Z=\left\{\pi_{\alpha}, \pi_{\beta}, \pi_{\gamma}, \pi_{\alpha}, \pi_{\beta}, \pi_{\gamma}\right\}$.

To determine the vanishing cycle one technically has to specify the order of the roots coming together, otherwise there is an ambiguity of sign. However, if a cycle vanishes at a singular point, so does its negative, and in the Picard-Lefschetz formula (2.4) the sign of the vanishing cycle has no effect on the local monodromy; therefore we will take the signs as indicated. Via successive application of the PicardLefschetz formula the monodromy around the entire set $Z$ is then computed to be $w_{S}(\lambda) \equiv w_{\pi_{\gamma}} \circ w_{\pi_{\beta}} \circ$ $w_{\pi_{\alpha}} \circ w_{\pi_{\gamma}} \circ w_{\pi_{\beta}} \circ w_{\pi_{\alpha}}(\lambda)=-\lambda$. This is precisely the monodromy of a $D_{4}$ singularity. However, the basis differs from the AAAABC case 19 .

Let us study string junctions using the ordered set of vanishing cycles $Z$. In this basis, the intersection product can be easily computed from equation (2.2). It is given by

$$
I=\left(\begin{array}{cccccc}
-1 & 1 / 2 & 1 / 2 & 0 & -1 / 2 & 1 / 2 \\
1 / 2 & -1 & -1 / 2 & 1 / 2 & 0 & -1 / 2 \\
1 / 2 & -1 / 2 & -1 & 1 / 2 & -1 / 2 & 0 \\
0 & 1 / 2 & 1 / 2 & -1 & 1 / 2 & -1 / 2 \\
-1 / 2 & 0 & -1 / 2 & 1 / 2 & -1 & 1 / 2 \\
1 / 2 & -1 / 2 & 0 & -1 / 2 & 1 / 2 & -1
\end{array}\right)
$$

There are 24 junctions $J \in \mathbb{Z}^{6}$ with $a(J)=\left(\begin{array}{l}0 \\ 0\end{array}\right)$ and $(J, J)=-2$. Call this set $R$. If $R$ are the roots of $D_{4}$, then there must exist 4-element subsets of $R$ which generate 12-element subsets of $R$ as positive linear combinations; that is, there must be simple roots which generate positive roots. The number of such sets must equal $\left|W_{D_{4}}\right|=2^{3} \cdot 4 !=192$, the order of the Weyl group of $D_{4}$. Direct computation shows that this is the case, and the results are presented in Table 10. Take the first of these sets to be the simple roots:

$$
\begin{array}{ll}
\alpha_{1}=(0,0,0,-1,-1,-1) & \alpha_{2}=(0,0,-1,0,0,1) \\
\alpha_{3}=(0,-1,0,0,1,0) & \alpha_{4}=(-1,0,1,1,0,-1) .
\end{array}
$$

In this Weyl Chamber, the highest root is given by the junction $J=(-1,-1,-1,0,0,0)$. A computation using Freudenthal's recursion formula (3.12) gives the correct level diagram.

Having established that junctions correctly give the $D_{4}$ algebra via the deformation (5.6) for a given $t$, let us now study the influence of encircling the locus $t=0$, parameterizing $t$ as $t=|t| e^{i \varphi}$. Clearly the depictions of the $z$-plane and $x$-plane above are identical at $\varphi$ a multiple of $2 \pi$, but varying $\varphi$ continuously between $2 \pi n$ and $2 \pi(n+1)$ could change the ordered set of vanishing cycles. Varying $\varphi$ between 0 and $2 \pi$, we see the action can be represented in the previous schematic diagram as follows:

\footnotetext{
${ }^{19}$ See the nice work 31 for an example obtaining $D_{4}$ from $G_{2}$ in the AAAABC basis. Unlike that work, however, the $\mathbb{Z}_{3}$ action we study is dictated by geometry and a different interpretation of the outer monodromy in terms of a codimension two locus.
} 


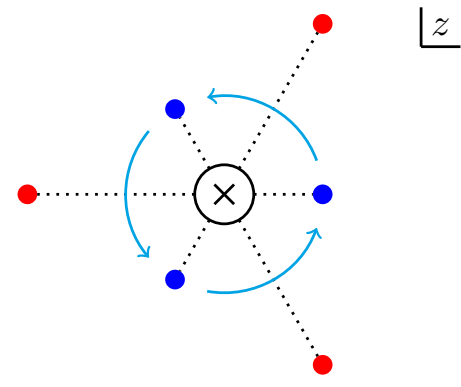

As $\varphi$ goes from 0 to $2 \pi$ the roots of $x^{3}+\epsilon t=0$ rotate through $\frac{2 \pi}{3}$, and the induced action on the torus above $P\left(y^{2}=x^{3}+\epsilon t\right)$ acts on homology by carrying $\pi_{\alpha}$ to $\pi_{\beta}, \pi_{\beta}$ to $\pi_{\gamma}$, and $\pi_{\gamma}$ back to $\pi_{\alpha}$. The three red points in the discriminant locus (in the $z$-plane) stay fixed, but the three blue ones rotate into one another in the same way, as indicated. By continuity, for each value $\arg t=\varphi$ (starting from zero and increasing), the two roots which determine $\pi_{\alpha}$ at $\varphi=0$ must continue to coalesce as $z$ moves from zero to the upper red point; however, $\varphi=2 \pi$ these same two points determine $\pi_{\beta}$ and therefore the vanishing cycle of the upper red dot is $\pi_{\beta}$. At the other two other red dots, going counter clockwise, the vanishing cycles are $\pi_{\alpha}$ and $\pi_{\gamma}$ for $\varphi=2 \pi$. On the other hand, the blue singular points move around (on a slightly off center circle for small $|t|$ ) in the same way as the roots in the $x$-plane, as $\varphi$ goes from zero to $2 \pi$. Therefore, starting with the first blue singular point to the left of the upper right red one and moving counter clockwise, we now get the vanishing cycles $\pi_{\gamma}, \pi_{\beta}$, and $\pi_{\alpha}$. Altogether, beginning again with the upper right red dot and moving counterclockwise, the ordered set of vanishing cycles at $\varphi=2 \pi$ is $Z_{2 \pi}=\left\{\pi_{\beta}, \pi_{\gamma}, \pi_{\alpha}, \pi_{\beta}, \pi_{\gamma}, \pi_{\alpha}\right\}$. Similarly, at $\varphi=4 \pi$ we have $Z_{4 \pi}=\left\{\pi_{\gamma}, \pi_{\alpha}, \pi_{\beta}, \pi_{\gamma}, \pi_{\alpha}, \pi_{\beta}\right\}$, and $Z_{0}=Z_{6 \pi}$, so that the effect of the monodromy of going around $t=0$ is to produce this permutation action of a cyclic group $\mathbb{Z}_{3}$ on the ordered set of vanishing cycles.

Given this action on the geometry we can determine the action on string junctions and therefore on the $D_{4}$ algebra. We will present two arguments which show the reduction of $G_{2}$ to $D_{4}$. First we will present a quick and straightforward argument using the same set of vectors in $\mathbb{Z}^{6}$, but in the different bases $Z_{\varphi}$; in this case the ordering in $Z$ begins with the upper red dot and moves counter-clockwise, with the vanishing cycles changing at different values of $\varphi$, and in this case the a junction $J$ emanating from the upper red dot would be $J=(1,0,0,0,0,0)$ for all three values of $\varphi$. Alternatively, we will present a second and more detailed argument where the first entry in $Z$ is the red dot with vanishing cycle $\pi_{a}$, and the rest follow by counterclockwise ordering. In this viewpoint, the same junction $J$ emanating from the upper red dot would be $J_{0}=(1,0,0,0,0,0), J_{2 \pi}=(0,0,0,0,1,0)$ and $J_{4 \pi}=(0,0,1,0,0,0)$ at the respective values of $\varphi$. In this case a matrix $T$ gives the action on junctions induced by monodromy around $t=0$.

We begin with the first approach. The three bases $Z_{0}, Z_{2 \pi}$, and $Z_{4 \pi}$ all have the same intersection product $I$, the same sets $R$, and all give rise to a $D_{4}$ algebra. For these reasons, any set $\left\{\beta_{1}, \beta_{2}, \beta_{3}, \beta_{4}\right\}$ of vectors in $\mathbb{Z}^{6}$ that determine simple roots at $\varphi=0$ also determine a set of simple roots at $\varphi=2 \pi, 4 \pi$. Since the Cartan matrix is identical in all three cases, the central node of the Dynkin diagram is fixed, but we must also determine if there is any action on the exterior nodes. We would first like to establish that the outer nodes of the Dynkin diagram rotate into one another under the monodromy around $t=0$. This can be done with a simple argument about the eight dimensional representations, which must be permuted by the automorphism. Junctions in the $8_{v}, 8_{s}$ and $8_{c}$ representations have $(J, J)=-1$ and $a(J)=\pi_{\alpha}$, $a(J)=\pi_{\beta}$ and $a(J)=\pi_{\gamma}$, respectively. For each phase $\varphi=0,2 \pi, 4 \pi$ of $t$, a junction ending on the upper 
red dot,

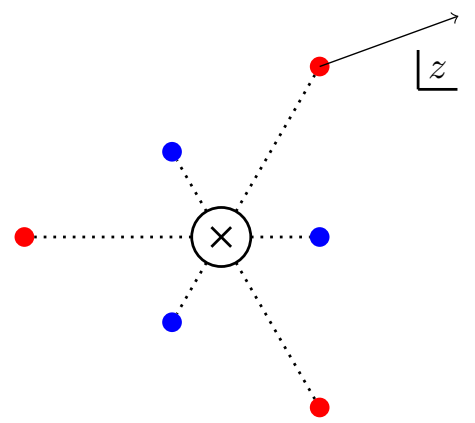

for example, has $(J, J)=-1$, but its asymptotic charge depends on $\varphi$. At $\varphi=0,2 \pi, 4 \pi$ this junction is therefore in the $8_{v}, 8_{s}, 8_{c}$ representation, respectively, giving the necessary triality permutation. But since the Dynkin labels of the highest weights of these representations are [87]

$$
8_{v}:(1,0,0,0) \quad 8_{s}:(0,0,0,1) \quad 8_{c}:(0,0,1,0),
$$

we see that an action permutes these representations if and only if it permutes the exterior nodes of the $D_{4}$ Dynkin diagram. Therefore we have the $\mathbb{Z}_{3}$ automorphism in (5.4), and the monodromy reduces the gauge symmetry from $D_{4}$ to $G_{2}$.

Though this argument is brief and correct, we will now use the second approach and see the direct action of monodromy on the junctions. This requires performing an analysis using $Z=Z_{0}$ and using the action on junctions given by the matrix

$$
T=\left(\begin{array}{llllll}
0 & 0 & 1 & 0 & 0 & 0 \\
0 & 0 & 0 & 1 & 0 & 0 \\
0 & 0 & 0 & 0 & 1 & 0 \\
0 & 0 & 0 & 0 & 0 & 1 \\
1 & 0 & 0 & 0 & 0 & 0 \\
0 & 1 & 0 & 0 & 0 & 0
\end{array}\right),
$$

which satisfies $T^{3}=1$. In this picture a junction emanating from the upper red dot is given by $(1,0,0,0,0,0),(0,0,0,0,1,0)$ and $(0,0,1,0,0,0)$ at $\varphi=0,2 \pi$, and $4 \pi$, respectively. Given the simple roots $\alpha_{i}$ as above, $T \alpha_{i}$ and $T^{2} \alpha_{i}$ give the simple roots at the corresponding $\varphi$; they are presented in Table 1 for convenience. It is clear that root junctions are not invariant, but in fact $T$ maps the simple roots to a completely different set of simple roots; we have moved to a different Weyl chamber. Automorphism requires that $\alpha_{2}, T \alpha_{2}$, and $T^{2} \alpha_{2}$ are the central node of their respective Dynkin diagrams. This is verified easily by computing the Cartan matrix at each $\varphi$. At any of these $\varphi$ the highest weights and next highest weights of the $8_{v}, 8_{s}$, and $8_{c}$ representations can be determined. Subtracting them gives a simple root, and it turns out that $\alpha_{4}, T \alpha_{4}$ and $T^{2} \alpha_{4}$ are the first simple roots subtracted from the $8_{v}, 8_{s}$, and $8_{c}$ representations at the respective $\varphi$ values. Similar statements hold for $\alpha_{1}$ and $\alpha_{3}$, proving that the three external nodes of the Dynkin are permuted by the $\mathbb{Z}^{3}$ action $T$. See Table 1 for more details. 
We have identified the appearance of the $D_{4}$ algebra and the $\mathbb{Z}_{3}$ O-monodromy around a codimension two locus that reduces it to $G_{2}$. This is evident in the level diagram for the adjoint of $D_{4}$

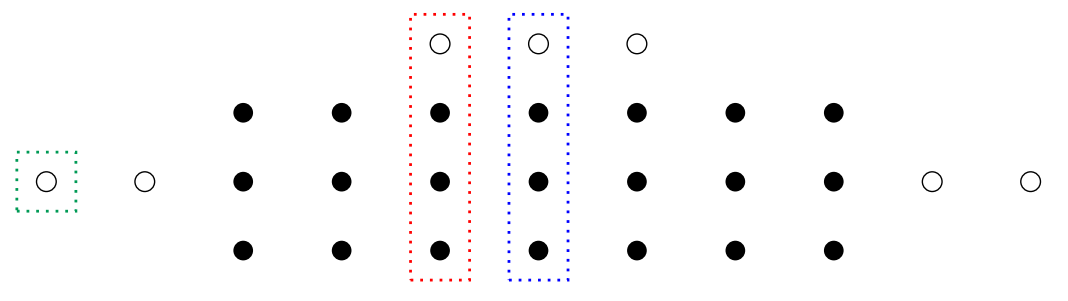

where the highest root is in a green box, the simple roots are in the red box, and the Cartan elements are in the blue box. In subtracting simple roots, one moves from left to right down the diagram. Any set of three black dots represents roots which permute into one another under the $\mathbb{Z}^{3}$ action; unfilled dots are invariant. This structure is clear in the Dynkin basis, where the highest weight is $(0,1,0,0)$ and the rest of the roots can be obtained by subtracting rows of the Cartan matrix in the usual way. For example $(0,1,0,0)$ is invariant under the permutation of the exterior simple roots $\alpha_{1} \rightarrow \alpha_{3}, \alpha_{3} \rightarrow \alpha_{4}, \alpha_{4} \rightarrow \alpha_{1}$; the roots at level 3 are $(0,1,0,0)-\alpha_{2}-\alpha_{i}$ for $i=1,3,4$, which also permute. Similar statements hold for junctions, with the caveat of the change in Weyl chamber, as discussed. Considering $\operatorname{span}_{\mathbb{Z}}\left(\alpha_{1}, \alpha_{3}, \alpha_{4}\right)$, the $\mathbb{Z}_{3}$ action on this space is induced by the matrix

$$
\left(\begin{array}{lll}
0 & 0 & 1 \\
1 & 0 & 0 \\
0 & 1 & 0
\end{array}\right)
$$

the eigenvalues of which are the three roots of unity, with the invariant subspace given by elements proportional to $\alpha_{1}+\alpha_{3}+\alpha_{4}$. The eigenvalues $e^{2 \pi i / 3}$ and $e^{4 \pi i / 3}$ have corresponding eigenvectors $\left(e^{4 \pi i / 3}, e^{2 \pi i / 3}, 1\right)$ and $\left(e^{2 \pi i / 3}, e^{4 \pi i / 3}, 1\right)$, respectively, with transpose implied when necessary. Similarly, each set of roots given by three black dots have an invariant subspace; together with unfilled dots, whose corresponding roots are $\mathbb{Z}_{3}$-invariant, there is a 14 -dimensional $\mathbb{Z}_{3}$-invariant subspace of the adjoint of $D_{4}$. This is the adjoint of $G_{2}$. Each set of three black dots contributes a two-dimensional non-invariant subspace, for a total of a 14 dimensional non-invariant subspace, which can be split into $7 \oplus 7$ according to the eigenvalues. In all, we have $\operatorname{Adj}\left(D_{4}\right)=28=\operatorname{Adj}\left(G_{2}\right) \oplus 7 \oplus 7=14 \oplus 7 \oplus 7$. At $t=0$ the monodromy ceases to act and M2-branes wrapped on the formerly non-invariant cycles give rise to a hypermultiplet in the 7 of $G_{2}$.

\subsection{Matter Representations in Particle Physics}

Many representations of ADE groups are considered in particle physics models, some of which are more natural than others. As emphasized throughout, the ADE representations realized via string junctions are broader than their weakly coupled type II counterparts. In this section we discuss phenomenologically relevant representations from the point of view of string junctions in the canonical basis (3.1). After recovering well-known facts, we discuss the realization of certain representations outside of the weak coupling limit, as well potential difficulties in realizing high dimensional representations in compact geometries. All Weyl chamber dependent statements in this section depend on the choice of simple roots given in Table 2 .

Consider $S U(5)$. The $\mathbf{5}$ is given by those junctions $J$ with $a(J)=(1,0)$ and $(J, J)=-1$; the $\overline{\mathbf{5}}$ by 


\begin{tabular}{c|c|c|c|c}
\hline$\varphi=0$ & $\alpha_{1}=(0,0,0,-1,-1,-1)$ & $\alpha_{2}=(0,0,-1,0,0,1)$ & $\alpha_{3}=(0,-1,0,0,1,0)$ & $\alpha_{4}=(-1,0,1,1,0,-1)$ \\
$\varphi=2 \pi$ & $T \alpha_{1}=(0,-1,-1,-1,0,0)$ & $T \alpha_{2}=(-1,0,0,1,0,0)$ & $T \alpha_{3}=(0,0,1,0,0,-1)$ & $T \alpha_{4}=(1,1,0,-1,-1,0)$ \\
$\varphi=4 \pi$ & $T^{2} \alpha_{1}=(-1,-1,0,0,0,-1)$ & $T^{2} \alpha_{2}=(0,1,0,0,-1,0)$ & $T^{2} \alpha_{3}=(1,0,0,-1,0,0)$ & $T^{2} \alpha_{4}=(0,-1,-1,0,1,1)$ \\
\hline
\end{tabular}

\begin{tabular}{r|c|c|c} 
& $\varphi=0$ & $\varphi=2 \pi$ & $\varphi=4 \pi$ \\
\hline Highest weight & $8_{v}:(-1,-1,0,1,0,-1)$ & $8_{s}:(0,1,0,-1,-1,-1)$ & $8_{c}:(0,-1,-1,-1,0,1)$ \\
Next highest weight & $8_{v}:(0,-1,-1,0,0,0)$ & $8_{s}:(-1,0,0,0,0,-1)$ & $8_{c}:(0,0,0,-1,-1,0)$ \\
Subtracted root & $\alpha_{4}$ & $T \alpha_{4}$ & $T^{2} \alpha_{4}$ \\
\hline Highest weight & $8_{c}:(-1,-1,0,0,0,0)$ & $8_{v}:(0,0,0,0,-1,-1)$ & $8_{s}:(0,0,-1,-1,0,0)$ \\
Next highest weight & $8_{c}:(-1,0,0,0,-1,0)$ & $8_{v}:(0,0,-1,0,-1,0)$ & $8_{s}:(-1,0,-1,0,0,0)$ \\
Subtracted root & $\alpha_{3}$ & $T \alpha_{3}$ & $T^{2} \alpha_{3}$ \\
\hline Highest weight & $8_{s}:(0,0,-1,-1,0,0)$ & $8_{c}:(-1,-1,0,0,0,0)$ & $8_{v}:(0,0,0,0,-1,-1)$ \\
Next highest weight & $8_{s}:(0,0,-1,0,1,1)$ & $8_{c}:(-1,0,1,1,0,0)$ & $8_{v}:(1,1,0,0,-1,0)$ \\
Subtracted root & $\alpha_{1}$ & $T \alpha_{1}$ & $T^{2} \alpha_{1}$ \\
\hline
\end{tabular}

Table 1: The top table gives the action of $T$ on each of the simple roots determined at $\varphi=0$, determining the simple roots at $\varphi=2 \pi, 4 \pi$. Note the Weyl chamber has changed. The first column of the bottom table gives the highest and next highest weight junction for each of the $8 d$ representations, together with the associated subtracted root. The data in the second and third columns are computed by $T$ and $T^{2}$ action.

$J$ with $a(J)=(-1,0)$ and $(J, J)=-1$. Their highest weights are given by $J_{5}=(1,0,0,0,0)$ and $J_{\overline{5}}=$ $(0,0,0,0,-1)$. From the type IIb point of view, the asymptotic charges signify that these representations are formed from fundamental strings coming out of and into stacks of D7-branes, respectively. The 10 is given by those $J$ with $a(J)=(2,0)$ and $(J, J)=-2$. The asymptotic charge $(2,0)$ demonstrates the $\mathbf{1 0}$ can end on a $B C$ pair. In the type IIb limit this becomes an O7-plane and the $\mathbf{1 0}$ is localized at a D7-O7 intersection, as is well-known from CFT quantization and other techniques. From Table 3 it is easy to see that the junction $(0,0,0,1,1)$ in the $\mathbf{1 0}$ can be interpreted as a branching from the simple root $(0,0,0,1,1,-1,-1)$ of $S O(10)$; this is the junction realization of brane unfolding.

Consider $S O(10)$. One generation of the quarks and leptons in the standard model, together with the right-handed neutrinos, embed into a single $\mathbf{1 6}$ of $S O(10)$. It can be realized by the set of junctions with $(J, J)=-1$ and $a(J)=(1,1)$. The highest weight is $J_{16}=(1,1,1,1,0,-2,-1)$. From the asymptotic charge $(1,1)$, we see it is a bound state of a D-string and F-string from the type IIb perspective, and therefore it does not occur at weak coupling. Consider $E_{6}$. The $\mathbf{2 7}$ is given by the set of junctions with $a(J)=(1,0)$ and $(J, J)=-1$. In contrast to the $\mathbf{1 6}$ of $S O(10)$, this representation has the correct asymptotic charge for it to end on a D7-brane; however, seven-branes with gauge symmetry $E_{6}$ cannot be realized at weak coupling.

Many other representations are considered in particle physics, frequently exotic particles beyond the standard model introduced to realize a phenomenological mechanism. For example, in $S O(10)$ GUT models this includes the $\mathbf{1 2 6}$ of $S O(10)$, introduced in [88] in order to account for small neutrino masses. It is often remarked that such high-dimensional representations are difficult to realize in compactifications of string theory, M-theory, or F-theory; they do not exist in weakly coupled theories with D-branes, and they cannot embed into an adjoint of $E_{8}$ in the heterotic string or F-theory. They also cannot be realized in the free field heterotic string [3, 4].

String junctions can realize a broader spectrum of possibilities. It can be checked via Freudenthal's formula that $J_{126}=2 J_{16}=(2,2,2,2,0,-4,-2)$ is the highest weight of the $\mathbf{1 2 6}$. However, we emphasize that though this gives necessary conditions for holomorphic curves to realize the 126, it does not mean 
that such curves are realized in compact Calabi-Yau varieties. As one considers higher dimensional representations the maximum $(J, J)$ for junctions in those representations goes up. This puts conditions on self-intersection numbers of holomorphic curves. For example, the $\mathbf{4 3 , 7 5 8}$ of $E_{6}$ has some junctions with $(J, J)=-18$, and it is unclear whether there exist manifolds with appropriate curves. For example, in elliptic $K 3$ the holomorphic curves $C$ satisfy $C \cdot C \geq-2$ and thus these higher representations cannot be realized. It would be interesting to study concretely whether bounds on $C \cdot C$ in more generic manifolds limit the representation theory. Perhaps there is a no-go in F-theory on theories with 126 of $S O(10)$ similar to known results in the heterotic string [3,4].

\section{Conclusions and Future Directions}

We have studied the appearance of Lie algebra representations in elliptically fibered Kähler varieties via the deformation of algebraic singularities. A concise list of results is given in the introduction.

There are many interesting possibilities for future work. Formally, our work is a mathematical analysis of elliptic fibrations which is independent of any particular application within string theory. The connection between the deformation theory of singularities and Lie theory has been studied extensively in the mathematics literature, with seminal contributions from Grothendieck, Brieskorn [27], and Arnol'd [22].

In this paper we focused on the realization of ADE representations on the $N$ deformed discriminant components and the relationship to Picard-Lefschetz theory. We also studied the occurrence of the non simply laced algebra $\mathfrak{g}_{2}$ from this point of view. We hope that the discussions and examples in this paper will shed light on this important subject.

We have also emphasized throughout that knowing the junction realization of an ADE representation in terms of codimension one data does not necessarily mean that the representation can be realized in a compact elliptic fibration, let alone as massless representation localized in codimension two. As we

have seen, ADE representations of high dimension have some junctions $J$ with self-intersection $(J, J)$ a large negative integer; as pointed out in [17,28] in the case of surfaces, holomorphic representatives have $(J, J) \geq-2$, which limits the allowed ADE flavor representations of BPS states in $d=4 \mathcal{N}=2$ theories on D3 probes. It would be interesting to study whether similar constraints exist for compactifications on higher dimensional elliptic fibrations, in particular $d=4 \mathcal{N}=1$ compactifications of F-theory on elliptically fibered Calabi-Yau fourfolds. At the very least, the realization of high dimensional ADE representations in these compactifications requires specializing to high codimension subloci in moduli space; singularities giving rise to them are not typically double points [11,13, 14].

Finally, we would again like to emphasize the advantages of deformations in F-theory compactifications: unlike the Kähler moduli of resolutions, the complex structure moduli of deformations exist in both the defining M-theory compactification and in the F-theory limit, and it is therefore the more physical description of gauge theoretic structure in F-theory. For example, in GUTs described by the breaking of a higher rank group such as $E_{8}$, the finite volume two-cycles of the Higgsed $W$-bosons arise from the deformation, not resolution. Though there has been recent progress in understanding F-theory compactifications via the Coulomb branch of the defining M-theory compactification, it would be advantageous to understand the same physics via deformation; this may also shed light on open problems in F-theory. 


\section{Acknowledgments}

We thank F. Chen, M. Cvetič, R. Donagi, L. Everett, P. Fendley, D. Klevers, V. Kumar, P. Langacker, and $\mathrm{H}$. Yu for useful conversations. We are particularly indebted to K. Dienes for discussion of related issues in the heterotic string; to G. Kane, R. Lu, and B. Zheng for related discussions on $G_{2}$ manifolds; and to D.R. Morrison for extensive conversations and comments. A.G. and J.H. thank Z. Guralnik and B. Ovrut, M. Cvetič and I. García-Etxebarria respectively for previous collaborations on string junctions. J.H. is supported by the National Science Foundation under Grant No. PHY11-25915. J.L.S. is supported by DARPA, fund no. 553700. is the Class of 1939 Professor in the School of Arts and Sciences of the University of Pennsylvania and gratefully acknowledges the generosity of the Class of 1939. A.G. and J.H. gratefully acknowledge the hospitality and support of the Simons Center for Geometry and Physics.

\section{A Appendices on ADE Algebras}

In this appendix we present aspects of junction realizations of ADE algebras which are used throughout the paper. These are by no means complete, but may serve as a useful reference for the reader.

Specifically, in this appendix we give:

- Sets of simple roots for $D_{4}, D_{5}, E_{6}, E_{7}$, and $E_{8}$ in the canonical basis (3.1).

- The positive roots of $E_{6}, E_{7}$, and $E_{8}$ in Table 7 , Table 8 and Table 9 ,

- Intersection matrices $\mathrm{S}$ for $D_{r}, E_{6}, E_{7}$, and $E_{8}$ in the canonical basis (3.1).

- Maps $F$ from junctions $\mathbb{Z}^{N}$ in the canonical basis (3.1) to weights in $\mathbb{Z}^{r}$ in the Dynkin basis.

- The 192 sets of simple roots for $D_{4}$ using the vanishing cycles $Z$ of section 5.2 ,

- An illustration of the branching of adjoints of $E_{6}$ and $S O(8)$ into irreps of $S O(10)$ and $S U(4)$. 


\begin{tabular}{|c|c|}
\hline Algebra & Simple Roots \\
\hline$D_{4}$ & $(0,1,-1,0,0,0) \quad(0,0,1,-1,0,0)$ \\
\hline$D_{5}$ & $\begin{array}{c}(1,-1,0,0,0,0,0) \quad(0,1,-1,0,0,0,0) \\
(0,0,0,1,-1,0,0) \quad(0,0,1,-1,0,0,0) \\
\end{array}$ \\
\hline$E_{6}$ & $\begin{array}{ccc}(1,-1,0,0,0,0,0,0) & (0,1,-1,0,0,0,0,0) & (0,0,1,-1,0,0,0,0) \\
(0,0,0,1,1,-1,-1,0) & (0,0,0,0,0,0,1,-1) & (0,0,0,1,-1,0,0,0)\end{array}$ \\
\hline$E_{7}$ & $\begin{array}{cc}(1,-1,0,0,0,0,0,0,0) & (0,1,-1,0,0,0,0,0,0) \quad(0,0,1,-1,0,0,0,0,0) \\
(0,0,0,1,-1,0,0,0,0) & (0,0,0,0,1,1,-1,-1,0) \\
(0,0,0,0,0,0,0,1,-1) & (0,0,0,0,1,-1,0,0,0)\end{array}$ \\
\hline$E_{8}$ & $\begin{array}{ccc}(1,-1,0,0,0,0,0,0,0,0) & (0,1,-1,0,0,0,0,0,0,0) & (0,0,1,-1,0,0,0,0,0,0) \\
(0,0,0,1,-1,0,0,0,0,0) & (0,0,0,0,1,-1,0,0,0,0) & (0,0,0,0,0,1,1,-1,-1,0) \\
(0,0,0,0,0,0,0,0,1,-1) & (0,0,0,0,0,1,-1,0,0,0)\end{array}$ \\
\hline
\end{tabular}

Table 2: Junctions which are simple roots for a number of algebras. All examples use the canonical basis (3.1).

$(1,1,0,0,-1,-1)$
$(1,0,1,0,-1,-1)$,
$(0,1,1,0,-1,-1),(1,0,0,1,-1,-1),(1,0,0,-1,0,0)$,
$(0,1,0,1,-1,-1),(0,1,0,-1,0,0),(1,0,-1,0,0,0)$,
$0,0,1,1,-1,-1),(0,1,-1,0,0,0),(0,0,1,-1,0,0),(1,-1,0,0,0,0)$,
$(0,0,0,0,0,0),(0,0,0,0,0,0),(0,0,0,0,0,0),(0,0,0,0,0,0)$,
$(0,0,-1,-1,1,1),(-1,1,0,0,0,0),(0,-1,1,0,0,0),(0,0,-1,1,0,0)$,
$(0,-1,0,-1,1,1),(-1,0,1,0,0,0),(0,-1,0,1,0,0)$,
$(0,-1,-1,0,1,1),(-1,0,0,-1,1,1),(-1,0,0,1,0,0)$
$(-1,0,-1,0,1,1)$
$(-1,-1,0,0,1,1)$.

Table 3: The weight diagram for the roots of $S O(8)$ in the $A A A A B C$ basis. Upon Higgsing to $S U(4)$, the roots which give rise to the $\mathbf{6}, \overline{\mathbf{6}}, \mathbf{1 5}$ and $\mathbf{1}$ of $S U(4)$ are colored in green, blue, red, and black, respectively.

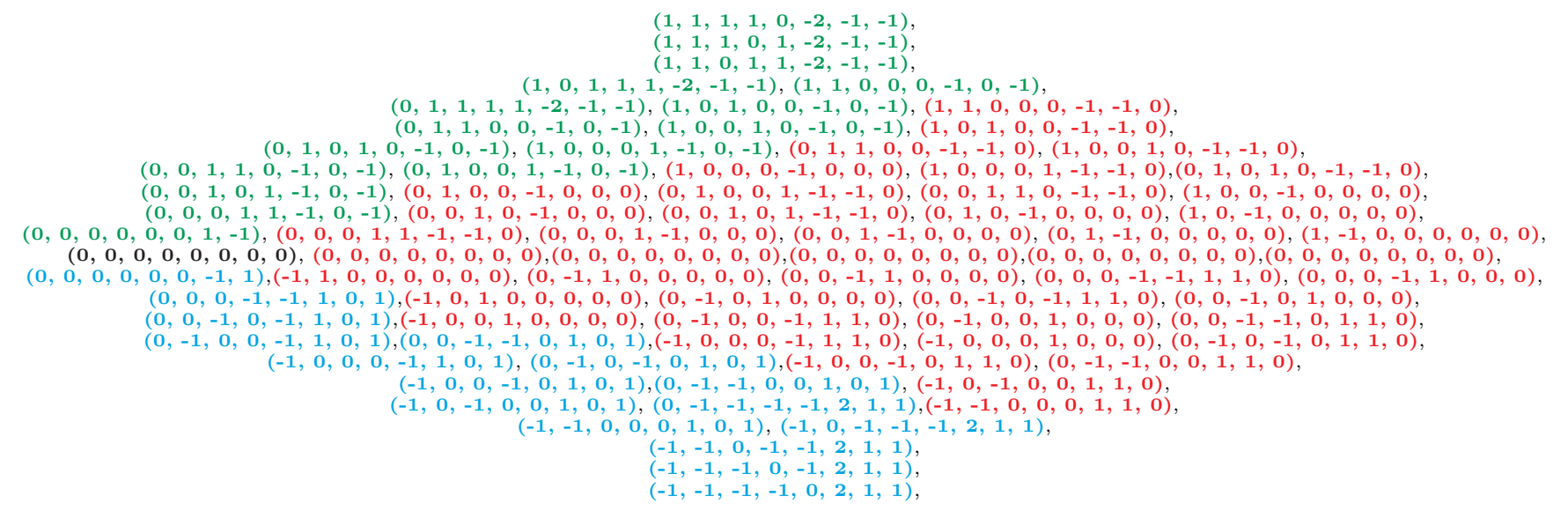

Table 4: The weight diagram for the roots of $E_{6}$ in the $A A A A A B C C$ basis. Upon Higgsing to $S O(10)$, the roots which give rise to the $\mathbf{1 6}, \mathbf{1 6}^{\prime}, \mathbf{4 5}$ and $\mathbf{1}$ of $S O(10)$ are colored in green, blue, red, and black, respectively. 


$$
I^{D_{r}}=\left(\begin{array}{ccccc}
-1 & \ldots & 0 & -\frac{1}{2} & \frac{1}{2} \\
0 & \ddots & 0 & \vdots & \vdots \\
0 & \cdots & -1 & -\frac{1}{2} & \frac{1}{2} \\
-\frac{1}{2} & \cdots & -\frac{1}{2} & -1 & 1 \\
\frac{1}{2} & \cdots & \frac{1}{2} & 1 & -1
\end{array}\right) \quad I^{E_{6}}=\left(\begin{array}{cccccccc}
-1 & 0 & 0 & 0 & 0 & -\frac{1}{2} & \frac{1}{2} & \frac{1}{2} \\
0 & -1 & 0 & 0 & 0 & -\frac{1}{2} & \frac{1}{2} & \frac{1}{2} \\
0 & 0 & -1 & 0 & 0 & -\frac{1}{2} & \frac{1}{2} & \frac{1}{2} \\
0 & 0 & 0 & -1 & 0 & -\frac{1}{2} & \frac{1}{2} & \frac{1}{2} \\
0 & 0 & 0 & 0 & -1 & -\frac{1}{2} & \frac{1}{2} & \frac{1}{2} \\
-\frac{1}{2} & -\frac{1}{2} & -\frac{1}{2} & -\frac{1}{2} & -\frac{1}{2} & -1 & 1 & 1 \\
\frac{1}{2} & \frac{1}{2} & \frac{1}{2} & \frac{1}{2} & \frac{1}{2} & 1 & -1 & 0 \\
\frac{1}{2} & \frac{1}{2} & \frac{1}{2} & \frac{1}{2} & \frac{1}{2} & 1 & 0 & -1
\end{array}\right)
$$

$I^{E_{7}}=\left(\begin{array}{ccccccccc}-1 & 0 & 0 & 0 & 0 & 0 & -\frac{1}{2} & \frac{1}{2} & \frac{1}{2} \\ 0 & -1 & 0 & 0 & 0 & 0 & -\frac{1}{2} & \frac{1}{2} & \frac{1}{2} \\ 0 & 0 & -1 & 0 & 0 & 0 & -\frac{1}{2} & \frac{1}{2} & \frac{1}{2} \\ 0 & 0 & 0 & -1 & 0 & 0 & -\frac{1}{2} & \frac{1}{2} & \frac{1}{2} \\ 0 & 0 & 0 & 0 & -1 & 0 & -\frac{1}{2} & \frac{1}{2} & \frac{1}{2} \\ 0 & 0 & 0 & 0 & 0 & -1 & -\frac{1}{2} & \frac{1}{2} & \frac{1}{2} \\ -\frac{1}{2} & -\frac{1}{2} & -\frac{1}{2} & -\frac{1}{2} & -\frac{1}{2} & -\frac{1}{2} & -1 & 1 & 1 \\ \frac{1}{2} & \frac{1}{2} & \frac{1}{2} & \frac{1}{2} & \frac{1}{2} & \frac{1}{2} & 1 & -1 & 0 \\ \frac{1}{2} & \frac{1}{2} & \frac{1}{2} & \frac{1}{2} & \frac{1}{2} & \frac{1}{2} & 1 & 0 & -1\end{array}\right)\left(\begin{array}{cccccccccc}-1 & 0 & 0 & 0 & 0 & 0 & 0 & -\frac{1}{2} & \frac{1}{2} & \frac{1}{2} \\ 0 & -1 & 0 & 0 & 0 & 0 & 0 & -\frac{1}{2} & \frac{1}{2} & \frac{1}{2} \\ 0 & 0 & -1 & 0 & 0 & 0 & 0 & -\frac{1}{2} & \frac{1}{2} & \frac{1}{2} \\ 0 & 0 & 0 & -1 & 0 & 0 & 0 & -\frac{1}{2} & \frac{1}{2} & \frac{1}{2} \\ 0 & 0 & 0 & 0 & -1 & 0 & 0 & -\frac{1}{2} & \frac{1}{2} & \frac{1}{2} \\ 0 & 0 & 0 & 0 & 0 & -1 & 0 & -\frac{1}{2} & \frac{1}{2} & \frac{1}{2} \\ 0 & 0 & 0 & 0 & 0 & 0 & -1 & -\frac{1}{2} & \frac{1}{2} & \frac{1}{2} \\ -\frac{1}{2} & -\frac{1}{2} & -\frac{1}{2} & -\frac{1}{2} & -\frac{1}{2} & -\frac{1}{2} & -\frac{1}{2} & -1 & 1 & 1 \\ \frac{1}{2} & \frac{1}{2} & \frac{1}{2} & \frac{1}{2} & \frac{1}{2} & \frac{1}{2} & \frac{1}{2} & 1 & -1 & 0 \\ \frac{1}{2} & \frac{1}{2} & \frac{1}{2} & \frac{1}{2} & \frac{1}{2} & \frac{1}{2} & \frac{1}{2} & 1 & 0 & -1\end{array}\right)$

Table 5: Intersection matrices for $D_{r}, E_{6}, E_{7}$, and $E_{8}$ in the canonical basis (2.2), computed from the generic formula (2.2). These match [15]. 


$$
\begin{aligned}
& F^{D_{4}}=\left(\begin{array}{ccccccc}
1 & -1 & 0 & 0 & 0 & 0 & 0 \\
0 & 1 & -1 & 0 & 0 & 0 & 0 \\
0 & 0 & 1 & -1 & 0 & 0 & 0 \\
0 & 0 & 0 & 1 & -1 & 0 & 0 \\
0 & 0 & 0 & 1 & 1 & 1 & -1
\end{array}\right) \quad F^{D_{5}}=\left(\begin{array}{ccccccc}
1 & -1 & 0 & 0 & 0 & 0 & 0 \\
0 & 1 & -1 & 0 & 0 & 0 & 0 \\
0 & 0 & 1 & -1 & 0 & 0 & 0 \\
0 & 0 & 0 & 1 & -1 & 0 & 0 \\
0 & 0 & 0 & 1 & 1 & 1 & -1
\end{array}\right) \\
& F^{E_{6}}=\left(\begin{array}{cccccccc}
1 & -1 & 0 & 0 & 0 & 0 & 0 & 0 \\
0 & 1 & -1 & 0 & 0 & 0 & 0 & 0 \\
0 & 0 & 1 & -1 & 0 & 0 & 0 & 0 \\
0 & 0 & 0 & 1 & 1 & 1 & -1 & 0 \\
0 & 0 & 0 & 0 & 0 & 0 & 1 & -1 \\
0 & 0 & 0 & 1 & -1 & 0 & 0 & 0
\end{array}\right) \quad F^{E_{7}}=\left(\begin{array}{ccccccccc}
1 & -1 & 0 & 0 & 0 & 0 & 0 & 0 & 0 \\
0 & 1 & -1 & 0 & 0 & 0 & 0 & 0 & 0 \\
0 & 0 & 1 & -1 & 0 & 0 & 0 & 0 & 0 \\
0 & 0 & 0 & 1 & -1 & 0 & 0 & 0 & 0 \\
0 & 0 & 0 & 0 & 1 & 1 & 1 & -1 & 0 \\
0 & 0 & 0 & 0 & 0 & 0 & 0 & 1 & -1 \\
0 & 0 & 0 & 0 & 1 & -1 & 0 & 0 & 0
\end{array}\right) \\
& F^{E_{8}}=\left(\begin{array}{cccccccccc}
1 & -1 & 0 & 0 & 0 & 0 & 0 & 0 & 0 & 0 \\
0 & 1 & -1 & 0 & 0 & 0 & 0 & 0 & 0 & 0 \\
0 & 0 & 1 & -1 & 0 & 0 & 0 & 0 & 0 & 0 \\
0 & 0 & 0 & 1 & -1 & 0 & 0 & 0 & 0 & 0 \\
0 & 0 & 0 & 0 & 1 & -1 & 0 & 0 & 0 & 0 \\
0 & 0 & 0 & 0 & 0 & 1 & 1 & 1 & -1 & 0 \\
0 & 0 & 0 & 0 & 0 & 0 & 0 & 0 & 1 & -1 \\
0 & 0 & 0 & 0 & 0 & 1 & -1 & 0 & 0 & 0
\end{array}\right)
\end{aligned}
$$

Table 6: In the canonical basis (3.1), these are maps $F: \mathbb{Z}^{N} \rightarrow \mathbb{Z}^{r}$ which map junctions to weights in the Dynkin basis. A general formula is given in section [3.5. These reproduce the maps of [15]. 


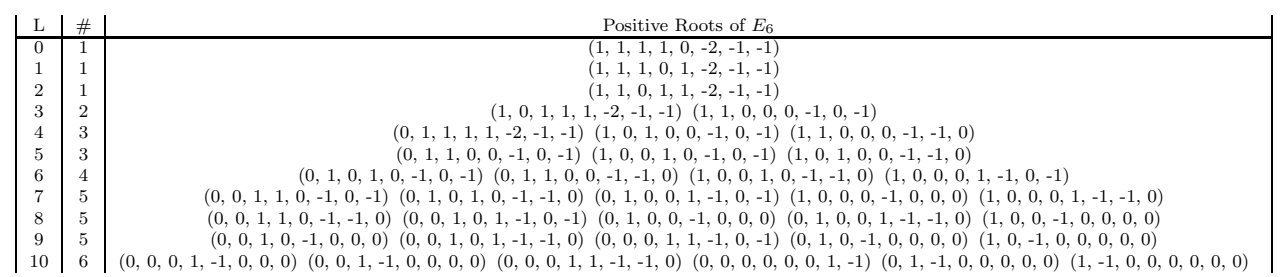

Table 7: Positive roots of $E_{6}$. Listed are the level $L$, the \# of roots at that level, and the roots themselves in the canonical junction basis (3.1).

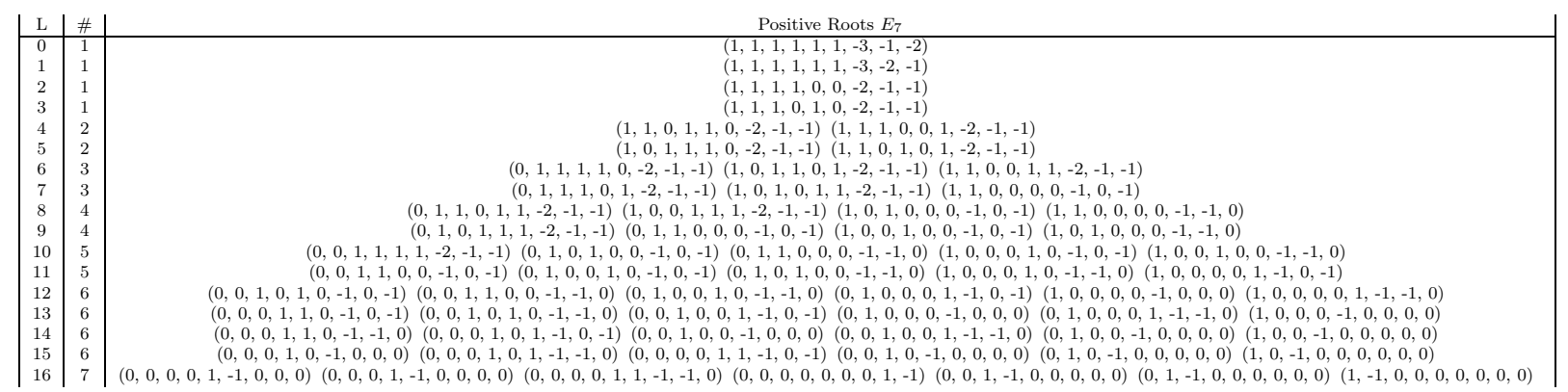

Table 8: Positive roots of $E_{7}$. Listed are the level $L$, the \# of roots at that level, and the roots themselves in the canonical junction basis (3.1).

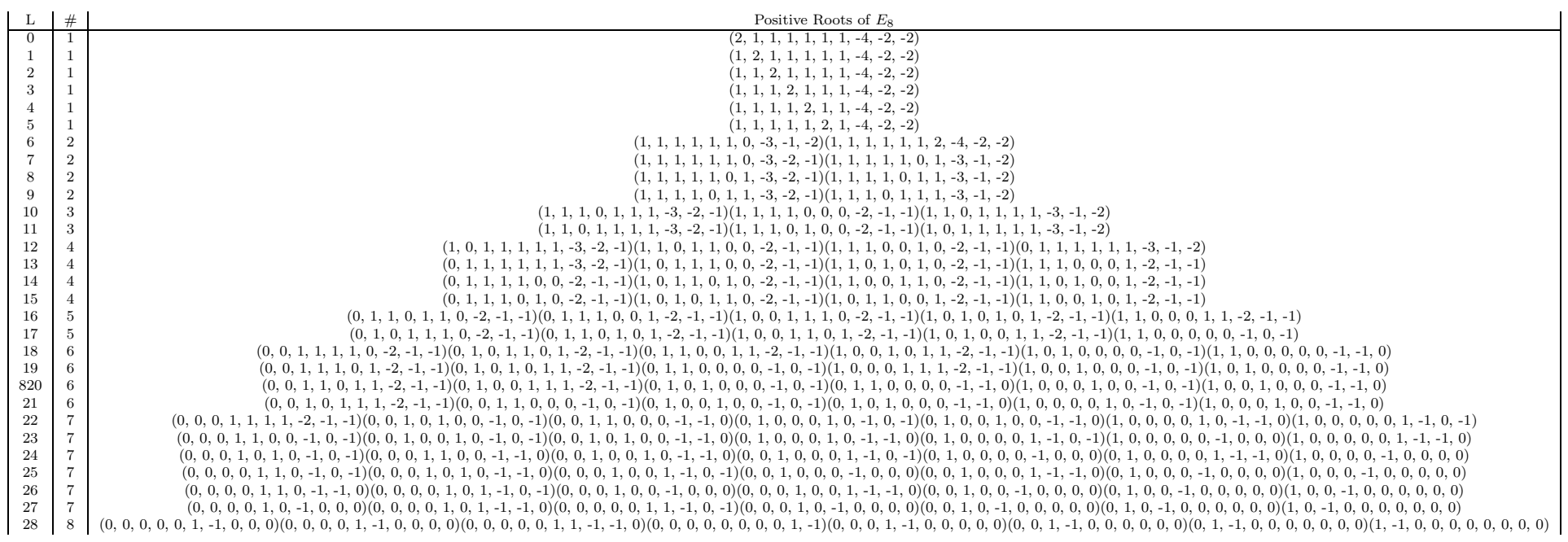

Table 9: Positive roots of $E_{8}$. Listed are the level $L$, the \# of roots at that level, and the roots themselves in the canonical junction basis ( 3.1 ). 


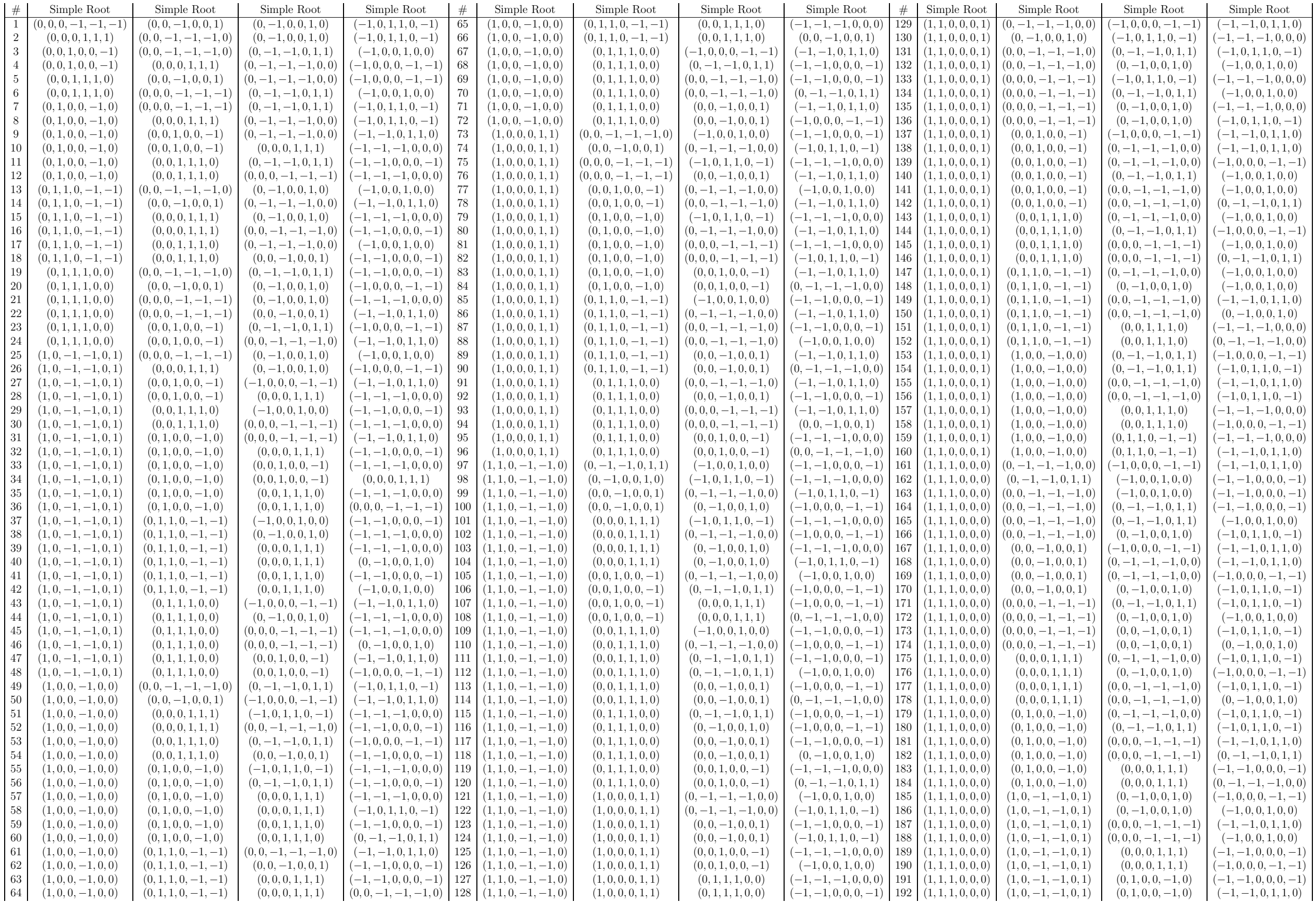

Table 10: The possible simple roots of $D_{4}$, represented in the basis $Z=\left\{\pi_{\alpha}, \pi_{\beta}, \pi_{\gamma}, \pi_{\alpha}, \pi_{\beta}, \pi_{\gamma}\right\}$ as discussed in section 5.2 . There are 192 possibilities, as there must be since the order of $W \operatorname{eyl}\left(D_{4}\right)=2^{3} \cdot 4$ !. We present this data for convenience and to demonstrate the agreement with the Weyl group. 


\section{B A Computational Package for Junctions}

Many of the computations in this paper are simple, though tedious. To facilitate computations, the authors have written a computer code which performs many of the relevant operations. This code is publicly available at

$$
\text { http://github.com/jhhalverson/py-junctions. }
$$

Though it is written in Python, it is best executed through a SAGE terminal, since it utilizes packages which are automatically included in SAGE. All non-trivial computations in this paper were performed using this package, and there are many example computations included on the web page. See also the Wiki which demonstrates many of the relevant methods. 


\section{Explicit Junction Depiction}

Figure 2 depicts a two-sphere wrapped by an M2-brane in the deformation picture of an M-theory compactification; in the F-theory limit this becomes a three-pronged string junction along a onemanifold in the F-theory base $\tilde{B}$.

Let's discuss the topology of this two-sphere. The green, blue, and red dots denote the points where the junction ends on seven-branes. The one-cycles $\pi_{x}, \pi_{y}$, and $-\pi_{x}-\pi_{y}$ vanish over each of these codimension one loci, respectively. The minus sign $-\pi_{x}-\pi_{y}$ corresponds to the orientation of this curve as a vanishing cycle for the red singular point, so that we get total asymptotic charge zero. The junction has one dimension along the elliptic fiber and one along $\tilde{B}$. Moving away from the green, blue, and red marked points, the vanishing cycle grow and are wrapped by one dimension of an M2-brane, depicted by the tori (represented as squares with periodic boundary conditions) with one-cycles in green, blue, and red.

At the junction point in $\tilde{B}$, the M2-brane wraps the one-cycle depicted by the square with both blue and green lines; the self-intersection point is evident. At this point the junction forms a pair of pants, and moving past the junction point the M2-brane wraps the purple cycle $\pi_{x}+\pi_{y}$. Continuing down the junction to points $\mathrm{A}, \mathrm{B}$, and $\mathrm{C}$ the cycle wrapped by the M2-brane moves towards the red cycle $-\pi_{x}-\pi_{y}$; somewhere between points $\mathrm{C}$ and $\mathrm{D}$, they merge, or more precisely in terms of the orientations determined as vanishing cycles, bound a common annulus, closing the two disks (with one and two marked points) into a sphere with three marked points. This sphere is the two-cycle wrapped by the M2-brane. 


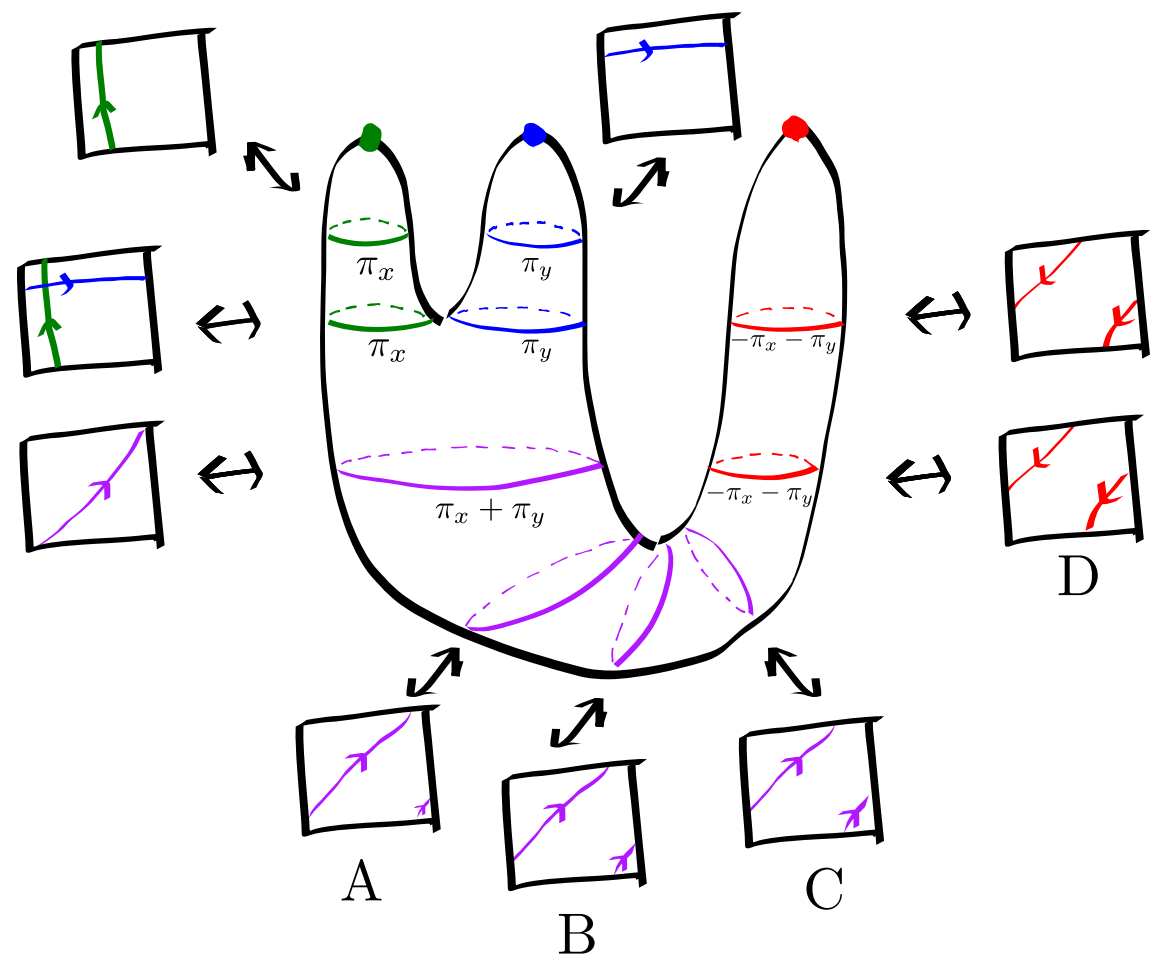

Figure 2: An explicit depiction of the topology of a three-pronged junction. See appendix C for an explicit description of this two-sphere. 


\section{References}

[1] E. Witten, "String Theory Dynamics in Various Dimensions," Nucl.Phys. B443 (1995) 85-126, hep-th/9503124.

[2] C. Vafa, "Evidence for F-Theory," Nucl. Phys. B469 (1996) 403-418, hep-th/9602022.

[3] K. R. Dienes and J. March-Russell, "Realizing Higher Level Gauge Symmetries in String Theory: New Embeddings for String GUTs," Nucl.Phys. B479 (1996) 113-172, hep-th/9604112.

[4] K. R. Dienes, "New Constraints on SO(10) Model Building from String Theory," Nucl.Phys. B488 (1997) 141-158, hep-ph/9606467.

[5] A. Kovalev, "Twisted connected sums and special Riemannian holonomy," ArXiv Mathematics e-prints (Dec., 2000) arXiv:math/0012189.

[6] A. Kovalev and N.-H. Lee, "K3 surfaces with non-symplectic involution and compact irreducible $G_{2}$-manifolds," ArXiv e-prints (Oct., 2008) 0810.0957.

[7] A. Corti, M. Haskins, J. Nordstrom, and T. Pacini, "Asymptotically cylindrical Calabi-Yau 3-folds from weak Fano 3-folds," ArXiv e-prints (June, 2012) 1206.2277.

[8] A. Corti, M. Haskins, J. Nordstrom, and T. Pacini, " $G_{2}$-manifolds and associative submanifolds via semi-Fano 3-folds," ArXiv e-prints (July, 2012) 1207.4470.

[9] S. H. Katz and C. Vafa, "Matter from Geometry," Nucl.Phys. B497 (1997) 146-154, hep-th/9606086.

[10] M. Bershadsky et al., "Geometric Singularities and Enhanced Gauge Symmetries," Nucl. Phys. B481 (1996) 215-252, hep-th/9605200.

[11] A. Grassi and D. R. Morrison, "Group Representations and the Euler Characteristic of Elliptically Fibered Calabi-Yau Threefolds," math/0005196.

[12] P. S. Aspinwall, S. H. Katz, and D. R. Morrison, "Lie Groups, Calabi-Yau Threefolds, and F Theory," Adv.Theor.Math.Phys. 4 (2000) 95-126, hep-th/0002012.

[13] D. R. Morrison and W. Taylor, "Matter and Singularities," JHEP 1201 (2012) 022, 1106.3563 .

[14] A. Grassi and D. R. Morrison, "Anomalies and the Euler Characteristic of Elliptic Calabi-Yau Threefolds," 1109.0042.

[15] O. DeWolfe and B. Zwiebach, "String Junctions for Arbitrary Lie Algebra Representations," Nucl. Phys. B541 (1999) 509-565, hep-th/9804210.

[16] M. R. Gaberdiel and B. Zwiebach, "Exceptional Groups from Open Strings," Nucl. Phys. B518 (1998) 151-172, hep-th/9709013.

[17] A. Mikhailov, N. Nekrasov, and S. Sethi, "Geometric Realizations of BPS States in $\mathcal{N}=2$ Theories," Nucl. Phys. B531 (1998) 345-362, hep-th/9803142. 
[18] D. R. Morrison and C. Vafa, "Compactifications of F-Theory on Calabi-Yau Threefolds - I," Nucl. Phys. B473 (1996) 74-92, hep-th/9602114.

[19] D. R. Morrison and C. Vafa, "Compactifications of F-Theory on Calabi-Yau Threefolds II," Nucl. Phys. B476 (1996) 437-469, hep-th/9603161.

[20] A. Grassi, J. Halverson, and J. Shaneson, "Resolution and Deformation of Elliptic Fibrations,".

[21] S. Katz and D. R. Morrison, "Gorenstein Threefold Singularities with Small Resolutions via Invariant Theory for Weyl Groups,".

[22] V. I. Arnol'd, "NORMAL FORMS OF FUNCTIONS IN NEIGHBOURHOODS OF DEGENERATE CRITICAL POINTS," Russian Mathematical Surveys 29 (1974), no. 2, 10.

[23] Gusein-Zade, S. M., "Monodromy groups of isolated singularities of hypersurfaces. (Russian)," Uspehi Mat. Nauk (1977).

[24] A. Hanany and E. Witten, "Type IIB Superstrings, BPS Monopoles, and Three- Dimensional Gauge Dynamics," Nucl. Phys. B492 (1997) 152-190, hep-th/9611230.

[25] R. Miranda, "Smooth models for elliptic threefolds," in The birational geometry of degenerations (Cambridge, Mass., 1981), vol. 29 of Progr. Math., pp. 85-133. Birkhäuser Boston, Mass., 1983.

[26] E. Witten, "Phase Transitions in M Theory and F Theory," Nucl.Phys. B471 (1996) 195-216, hep-th/9603150.

[27] E. Brieskorn, "Singular elements of semi-simple algebraic groups,".

[28] O. DeWolfe, T. Hauer, A. Iqbal, and B. Zwiebach, "Constraints on the BPS Spectrum of $\mathcal{N}=2, \mathrm{D}=4$ Theories with A-D-E Flavor Symmetry," Nucl. Phys. B534 (1998) 261-274, hep-th/9805220.

[29] A. Grassi, Z. Guralnik, and B. A. Ovrut, "Five-brane BPS states in heterotic M theory," JHEP 0101 (2001) 037, hep-th/0005121.

[30] A. Grassi, Z. Guralnik, and B. A. Ovrut, "Knots, braids and BPS states in M theory," JHEP 0206 (2002) 023, hep-th/0110036.

[31] L. Bonora and R. Savelli, "Non-Simply-Laced Lie Algebras via F Theory Strings," JHEP 1011 (2010) 025, 1007.4668.

[32] R. Donagi and M. Wijnholt, "Model Building with F-Theory," 0802.2969.

[33] C. Beasley, J. J. Heckman, and C. Vafa, "Guts and Exceptional Branes in F-Theory - I," JHEP 01 (2009) 058, 0802.3391.

[34] B. Andreas and G. Curio, "From Local to Global in F-Theory Model Building," J.Geom.Phys. 60 (2010) 1089-1102, 0902.4143. 
[35] J. Marsano, N. Saulina, and S. Schafer-Nameki, "F-Theory Compactifications for Supersymmetric Guts," JHEP 08 (2009) 030, 0904.3932.

[36] A. Collinucci, "New F-Theory Lifts II: Permutation Orientifolds and Enhanced Singularities," JHEP 04 (2010) 076, 0906.0003.

[37] R. Blumenhagen, T. W. Grimm, B. Jurke, and T. Weigand, "F-Theory Uplifts and GUTs," JHEP 09 (2009) 053, 0906.0013.

[38] J. Marsano, N. Saulina, and S. Schafer-Nameki, "Monodromies, Fluxes, and Compact Three-Generation F-Theory GUTs," JHEP 08 (2009) 046, 0906.4672.

[39] R. Blumenhagen, T. W. Grimm, B. Jurke, and T. Weigand, "Global F-Theory GUTs," Nucl. Phys. B829 (2010) 325-369, 0908.1784.

[40] J. Marsano, N. Saulina, and S. Schafer-Nameki, "Compact F-Theory Guts with $U(1)_{P Q}$," JHEP 04 (2010) 095, 0912.0272.

[41] T. W. Grimm, S. Krause, and T. Weigand, "F-Theory GUT Vacua on Compact Calabi-Yau Fourfolds," JHEP 07 (2010) 037, 0912.3524.

[42] M. Cvetič, I. García-Etxebarria, and J. Halverson, "Global F-Theory Models: Instantons and Gauge Dynamics," JHEP 1101 (2011) 073, 1003.5337.

[43] C.-M. Chen, J. Knapp, M. Kreuzer, and C. Mayrhofer, "Global SO(10) F-Theory Guts," JHEP 10 (2010) 057, 1005.5735.

[44] C.-M. Chen and Y.-C. Chung, "Flipped $S U(5)$ Guts from $E_{8}$ Singularities in F-Theory," JHEP 03 (2011) 049, 1005.5728.

[45] Y.-C. Chung, "On Global Flipped SU(5) Guts in F-Theory," JHEP 03 (2011) 126, 1008.2506 .

[46] C.-M. Chen and Y.-C. Chung, "On F-Theory E 6 Guts," JHEP 03 (2011) 129, 1010.5536.

[47] J. Knapp, M. Kreuzer, C. Mayrhofer, and N.-O. Walliser, "Toric Construction of Global F-Theory Guts," JHEP 03 (2011) 138, 1101.4908.

[48] J. Knapp and M. Kreuzer, "Toric Methods in F-Theory Model Building," Adv.High Energy Phys. 2011 (2011) 513436, 1103.3358.

[49] J. Marsano, H. Clemens, T. Pantev, S. Raby, and H.-H. Tseng, "A Global SU(5) F-Theory Model with Wilson Line Breaking," 1206.6132.

[50] T. W. Grimm and T. Weigand, "On Abelian Gauge Symmetries and Proton Decay in Global F- Theory Guts," Phys. Rev. D82 (2010) 086009, 1006.0226.

[51] M. J. Dolan, J. Marsano, N. Saulina, and S. Schafer-Nameki, "F-Theory Guts with U(1) Symmetries: Generalities and Survey," Phys.Rev. D84 (2011) 066008, 1102.0290.

[52] J. Marsano, N. Saulina, and S. Schafer-Nameki, "On G-Flux, M5 Instantons, and U(1)s in F-Theory," 1107.1718. 
[53] T. W. Grimm, M. Kerstan, E. Palti, and T. Weigand, "Massive Abelian Gauge Symmetries and Fluxes in F-Theory," JHEP 1112 (2011) 004, 1107.3842.

[54] D. R. Morrison and D. S. Park, "F-Theory and the Mordell-Weil Group of Elliptically-Fibered Calabi-Yau Threefolds," 1208.2695.

[55] J. Borchmann, C. Mayrhofer, E. Palti, and T. Weigand, "Elliptic Fibrations for $S U(5) \times$ $\mathrm{U}(1) \times \mathrm{U}(1)$ F-Theory Vacua," 1303.5054.

[56] M. Cvetič, D. Klevers, and H. Piragua, "F-Theory Compactifications with Multiple U(1)-Factors: Constructing Elliptic Fibrations with Rational Sections," 1303.6970.

[57] R. Blumenhagen, A. Collinucci, and B. Jurke, "On Instanton Effects in F-Theory," JHEP 08 (2010) 079, 1002.1894.

[58] R. Donagi and M. Wijnholt, "MSW Instantons," 1005.5391.

[59] T. W. Grimm, M. Kerstan, E. Palti, and T. Weigand, "On Fluxed Instantons and Moduli Stabilisation in IIB Orientifolds and F-Theory," 1105.3193.

[60] M. Cvetic, I. Garcia Etxebarria, and J. Halverson, "Three Looks at Instantons in F-theory New Insights from Anomaly Inflow, String Junctions and Heterotic Duality," JHEP 111 (2011) 101, 1107.2388.

[61] M. Bianchi, A. Collinucci, and L. Martucci, "Magnetized E3-Brane Instantons in F-Theory," JHEP 1112 (2011) 045, 1107.3732.

[62] M. Kerstan and T. Weigand, "Fluxed M5-Instantons in F-Theory," 1205.4720.

[63] M. Cvetič, R. Donagi, J. Halverson, and J. Marsano, "On Seven-Brane Dependent Instanton Prefactors in F-Theory," JHEP 1211 (2012) 004, 1209.4906.

[64] M. Bianchi, G. Inverso, and L. Martucci, "Brane Instantons and Fluxes in F-Theory," 1212.0024.

[65] J. Marsano, N. Saulina, and S. Schafer-Nameki, "A Note on G-Fluxes for F-Theory Model Building," JHEP 11 (2010) 088, 1006.0483.

[66] A. Collinucci and R. Savelli, "On Flux Quantization in F-Theory," JHEP 1202 (2012) 015, 1011.6388 .

[67] A. P. Braun, A. Collinucci, and R. Valandro, "G-Flux in F-Theory and Algebraic Cycles," Nucl.Phys. B856 (2012) 129-179, 1107.5337.

[68] J. Marsano and S. Schafer-Nameki, "Yukawas, G-Flux, and Spectral Covers from Resolved Calabi- Yau's," JHEP 11098 (2011), arXiv:1108.1794.

[69] S. Krause, C. Mayrhofer, and T. Weigand, " $G_{4}$ Flux, Chiral Matter and Singularity Resolution in F-Theory Compactifications," Nucl.Phys. B858 (2012) 1-47, 1109.3454.

[70] T. W. Grimm and H. Hayashi, "F-Theory Fluxes, Chirality and Chern-Simons Theories," 1111.1232. 
[71] A. P. Braun, A. Collinucci, and R. Valandro, "Algebraic Description of G-Flux in F-Theory: New Techniques for F-Theory Phenomenology," 1202.5029.

[72] M. Kuntzler and S. Schafer-Nameki. "G-Flux and Spectral Divisors," arXiv 1205.5688.

[73] C. Lawrie and S. Schfer-Nameki, JHEP 1304, 061 (2013) [arXiv:1212.2949 [hep-th]].

[74] S. Krause, C. Mayrhofer, and T. Weigand, "Gauge Fluxes in F-Theory and Type IIB Orientifolds," JHEP 1208 (2012) 119, 1202.3138.

[75] A. Collinucci and R. Savelli, "On Flux Quantization in F-Theory II: Unitary and Symplectic Gauge Groups," JHEP 1208 (2012) 094, 1203.4542.

[76] J. Marsano, N. Saulina, and S. Schafer-Nameki, "Global Gluing and G-Flux," 1211.1097.

[77] V. Kumar, D. R. Morrison, and W. Taylor, "Mapping 6D $\mathcal{N}=1$ Supergravities to F-Theory," JHEP 02 (2010) 099, 0911.3393.

[78] V. Kumar, D. R. Morrison, and W. Taylor, "Global Aspects of the Space of 6D $\mathcal{N}=1$ Supergravities," JHEP 1011 (2010) 118, 1008.1062.

[79] V. Kumar, D. S. Park, and W. Taylor, "6D Supergravity without Tensor Multiplets," JHEP 1104 (2011) 080, 1011.0726.

[80] D. R. Morrison and W. Taylor, "Classifying Bases for 6D F-Theory Models," Central Eur.J.Phys. 10 (2012) 1072-1088, 1201.1943.

[81] M. Esole and S.-T. Yau, "Small Resolutions of SU(5)-models in F-Theory," 1107.0733.

[82] M. Cvetič, T. W. Grimm, and D. Klevers, "Anomaly Cancellation and Abelian Gauge Symmetries in F-Theory," 1210.6034.

[83] A. Sen, "F-Theory and Orientifolds," Nucl. Phys. B475 (1996) 562-578, hep-th/9605150.

[84] A. Sen, "Orientifold Limit of F-Theory Vacua," Phys. Rev. D55 (1997) 7345-7349, hep-th/9702165.

[85] N. Seiberg and E. Witten, "Monopole Condensation, and Confinement in $\mathcal{N}=2$ Supersymmetric Yang-Mills Theory," Nucl. Phys. B426 (1994) 19-52, hep-th/9407087.

[86] T. Banks, M. R. Douglas, and N. Seiberg, "Probing F-Theory with Branes," Phys. Lett. B387 (1996) 278-281, hep-th/9605199.

[87] R. Slansky, "Group Theory for Unified Model Building," Phys.Rept. 79 (1981) 1-128.

[88] K. Babu and R. Mohapatra, "Predictive Neutrino Spectrum in Minimal SO(10) Grand Unification," Phys.Rev.Lett. 70 (1993) 2845-2848, hep-ph/9209215. 